\title{
Risk Assessment of Energy-Efficient Walls
}

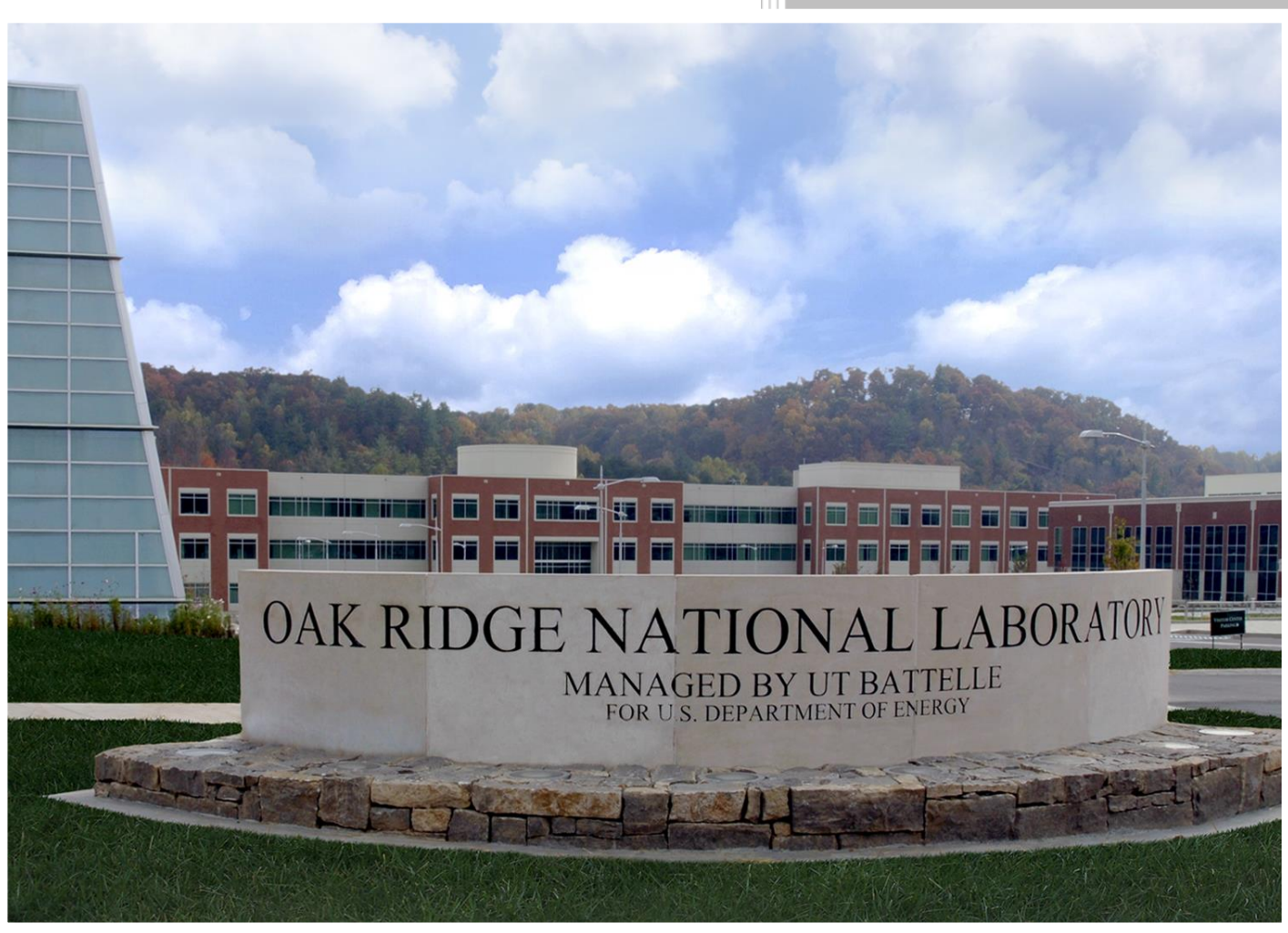

Approved for public release; distribution is unlimited.
Simon B. Pallin

Diana E. Hun Roderick K. Jackson Manfred Kehrer

December 2014 


\title{
DOCUMENT AVAILABILITY
}

Reports produced after January 1, 1996, are generally available free via US Department of Energy (DOE) SciTech Connect.

Website http://www.osti.gov/scitech/

Reports produced before January 1, 1996, may be purchased by members of the public from the following source:

\author{
National Technical Information Service \\ 5285 Port Royal Road \\ Springfield, VA 22161 \\ Telephone 703-605-6000 (1-800-553-6847) \\ TDD 703-487-4639 \\ Fax 703-605-6900 \\ E-mail info@ntis.gov \\ Website http://www.ntis.gov/help/ordermethods.aspx
}

Reports are available to DOE employees, DOE contractors, Energy Technology Data Exchange representatives, and International Nuclear Information System representatives from the following source:

Office of Scientific and Technical Information

PO Box 62

Oak Ridge, TN 37831

Telephone 865-576-8401

Fax 865-576-5728

E-mail reports@osti.gov

Website http://www.osti.gov/contact.html

This report was prepared as an account of work sponsored by an agency of the United States Government. Neither the United States Government nor any agency thereof, nor any of their employees, makes any warranty, express or implied, or assumes any legal liability or responsibility for the accuracy, completeness, or usefulness of any information, apparatus, product, or process disclosed, or represents that its use would not infringe privately owned rights. Reference herein to any specific commercial product, process, or service by trade name, trademark, manufacturer, or otherwise, does not necessarily constitute or imply its endorsement, recommendation, or favoring by the United States Government or any agency thereof. The views and opinions of authors expressed herein do not necessarily state or reflect those of the United States Government or any agency thereof. 
Energy and Transportation Science Division

Risk Assessment of Energy-Efficient Walls

\author{
Simon B. Pallin \\ Diana E. Hun \\ Roderick K. Jackson \\ Manfred Kehrer
}

Date Published: December 2014

\author{
Prepared by \\ OAK RIDGE NATIONAL LABORATORY \\ Oak Ridge, Tennessee 37831-6283 \\ managed by \\ UT-BATTELLE, LLC \\ for the \\ US DEPARTMENT OF ENERGY \\ under contract DE-AC05-00OR22725
}





\section{CONTENTS}

CONTENTS

Page

LIST OF FIGURES

iii

LIST OF TABLES $\mathrm{V}$

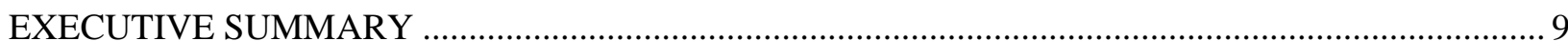

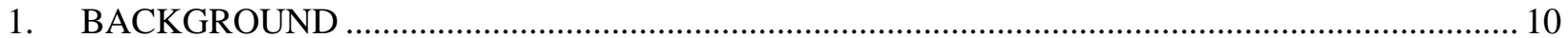

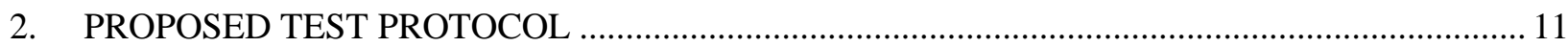

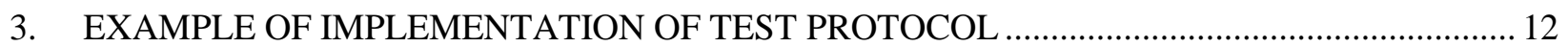

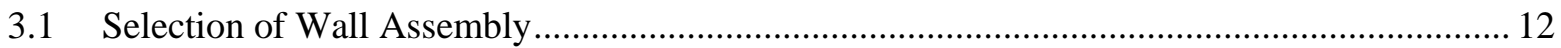

3.2 Identification of Potential Moisture Durability Problems ..................................................... 12

3.2.1 Expected Hygrothermal Performance and Potential Risks ....................................... 12

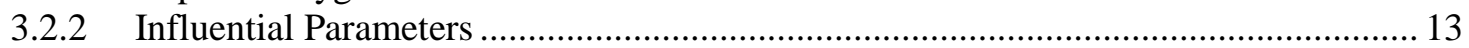

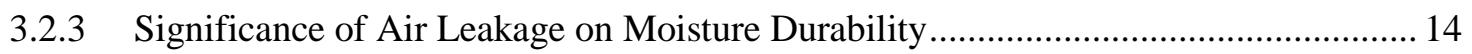

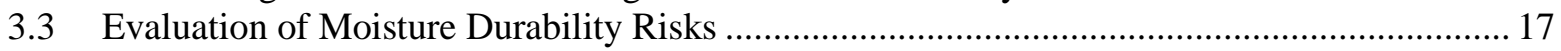

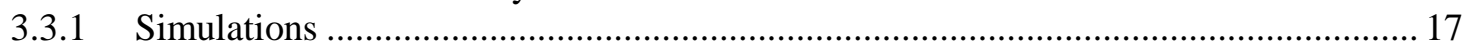

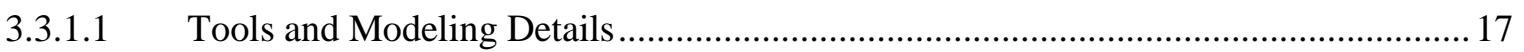

3.3.1.2 Calibration of the Hygrothermal Model for Air Leakage - The $\eta$-Method .................. 18

3.3.1.3 Initial Validation of Calibrated Simulation Tool ....................................................20

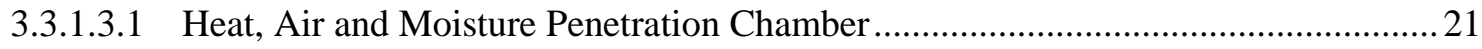

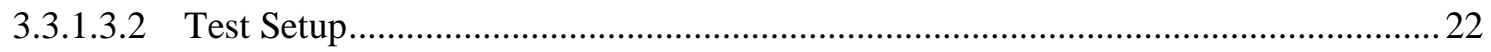

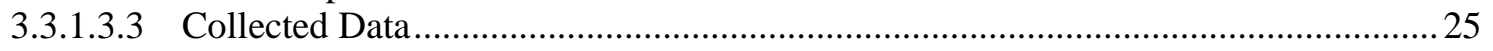

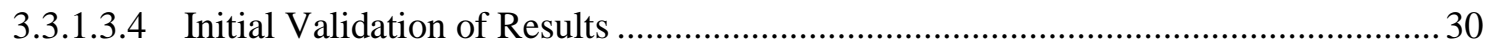

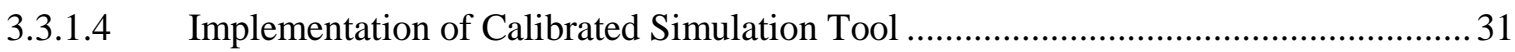

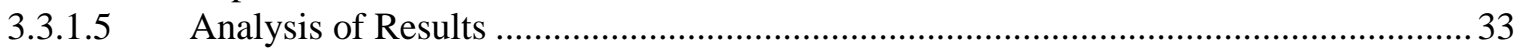

3.3.2 Validation of Simulations through Laboratory and Field Tests ................................. 39

3.4 Recommendations Based on Expected Hygrothermal Performances ..................................... 39

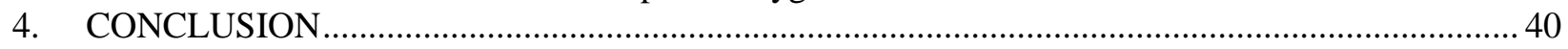

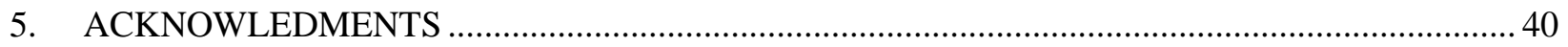

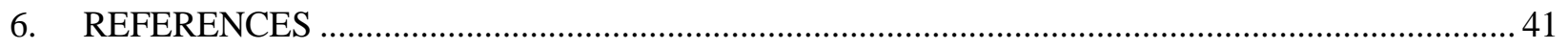

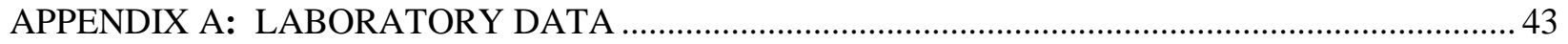

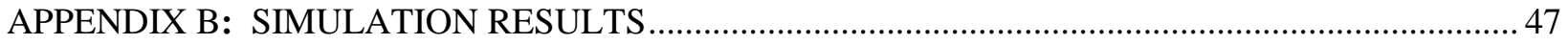





\section{LIST OF FIGURES}

Figure 1. Example of probability density distribution of moisture durability problems in walls. .......................11

Figure 2. Proposed moisture durability test protocol..........................................................................................12

Figure 3. Theoretical steady-state hygrothermal conditions of air inside a leakage path at different flow rates.

Figure 4. Steady-state hygrothermal conditions along an air leakage path after a step change in flow rate...16

Figure 5. Mold growth index as a function of exfiltration rates in a cold climate. ..............................................17

Figure 6. Calculated vs. simplified steady-state solutions for heat exchange in an air channel...........................19

Figure 7. Heat, air and moisture penetration chamber.................................................................................................21

Figure 8. Potential exfiltration and infiltration airflow paths through exterior walls...........................................22

Figure 9. Material assembly in test wall, and evaluated and actual airflow path...................................................23

Figure 10. Airflow path between fiberglass insulation and OSB, and insulation and top plate. .......................24

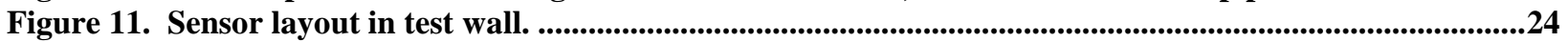

Figure 12. Followed sequence of indoor-to-outdoor pressure differentials across the test wall. ..........................25

Figure 13. Conditions in the indoor room (IR) and outdoor room (OR),..............................................................26

Figure 14. Pressure differentials across the test wall and flow rates through the test wall...................................26

Figure 15. Temperature along the airflow path in Wall Cavities $A$ and $B$........................................................27

Figure 16. Relative humidity along the airflow path in Wall Cavities $A$ and $B$. .................................................28

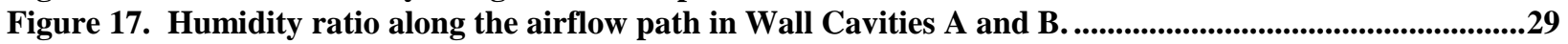

Figure 18. Simulated vs. measured steady-state temperatures at a leakage rate of $9.1 \mathrm{cfm}(4.3 \mathrm{~L} / \mathrm{s})$...............30

Figure 19. Simulated vs. measured stead-state humidity ratio levels at a leakage rate of $9.1 \mathrm{cfm}(4.3 \mathrm{~L} / \mathrm{s})$....31

Figure 20. Calculated vs. measured steady-state temperatures at a leakage rate of $5.8 \mathrm{cfm}(2.7 \mathrm{~L} / \mathrm{s})$..............31

Figure 21. Air changes per hour at a pressure differential of 50 Pa (ACH50) in Climate Zones 2 and 5 (LBL

2014). Specific levels of ACH50 are given for every tenth percentile. ...........................................................32

Figure 22. Locations where hygrothermal conditions were evaluated in Climate Zones 2 and 5.......................34

Figure 23. Relative humidity at OSB near the air's entry point in a wall in Climate Zone 5 and with high indoor moisture sources. The curves represent the results from a simulated wall with four different airtightness values (ACH50).

Figure 24. Relative humidity at OSB near the air's exit point in a wall in Climate Zone 5 and with high indoor moisture sources. The curves represent the results from a simulated wall with four different airtightness values (ACH50).

Figure 25. Relative humidity at drywall near the air's entry point in a wall in Climate Zone 2 and with high indoor moisture sources. The curves represent the results from a simulated wall with four different airtightness values (ACH50).

Figure 26. Relative humidity at drywall near the air's exit point in a wall in Climate Zone 2 and with high indoor moisture sources. The curves represent the results from a simulated wall with four different airtightness values (ACH50).

Figure 27. Mold growth index at OSB near the air's entry point in a wall in Climate Zone 5 and with a high indoor moisture supply.

Figure 28. Mold growth index at OSB near the air's exit point in a wall in Climate Zone 5 and with a high indoor moisture supply.

Figure 29. Mold growth index at drywall near the air's entry point in a wall in Climate Zone 2 and with a high indoor moisture supply.

Figure 30. Mold growth index at drywall near the air's exit point in a wall in Climate Zone 2 and with high a indoor moisture supply.

Figure 31. Mold growth index as a function of ACH50. 39 



\section{LIST OF TABLES}

Page

Table 1. Mold growth index per Hukka and Viitanen (1999) and Ojanen et al. (2010).....................................13

Table 2. Parameters controlled in the heat, air and moisture penetration chamber ............................................21

Table 3. Sensors. ........................................................................................................................................................21

Table 4. Average conditions in the indoor and outdoor rooms...................................................................................25 



\section{EXECUTIVE SUMMARY}

Walls in residential buildings are responsible for about 1.8 quads of the energy consumed in the US. This energy penalty can be reduced with higher insulation levels and increased airtightness; however, these measures can compromise the moisture durability and long-term performance of wall assemblies. Consequently, the design of energy-efficient walls needs to be accompanied by moisture risk assessments in order to ensure the realization of the expected decreases in energy use, accelerate their adoption rate, and build confidence within the construction industry.

This multi-year project aims to provide the residential construction industry with energy-efficient wall designs that are moisture durable. The present work focused on the initial step of this project, which is to develop a moisture durability protocol that identifies energy efficient wall designs that have a low probability of experiencing moisture problems. The proposed protocol:

- Examines the effects of moisture sources in walls through a combination of simulations, lab experiments and field tests;

- Calibrates a hygrothermal simulation tool so it can adequately model the effects of air leakage in wall assemblies;

- Is applicable to both retrofits and new constructions;

- Validates the calibrated model with laboratory and field tests; and

- Is based on a probabilistic approach that utilizes stochastically varying input parameters to expand the credibility and applicability of the results.

The present work also assessed the feasibility of the proposed protocol by conducting an example. $\mathrm{WUFI}^{\circledR} 2 \mathrm{D}$ was selected as the hygrothermal simulation tool, and was calibrated through a mathematical method. Thereafter, the calibrated WUFI ${ }^{\circledR} 2 \mathrm{D}$ was validated with laboratory measurements from a test wall that had a predefined air leakage path. Although the results were encouraging with regard to the hygrothermal effects of air leakage, more validation experiments will be performed. The calibrated WUFI $^{\circledR} 2 \mathrm{D}$ was also used to conduct a probabilistic risk assessment of a wall assembly in Climate Zones 2 and 5. This analysis was used to determine the critical $\left(Q_{c r}\right)$ or maximum allowed air leakage rate, where walls with leakage rates that are lower than $Q_{c r}$ have a low probability of experiencing mold growth. The critical air leakage rate will serve as a mechanism to compare the moisture tolerance of various wall assemblies.

As part of this research activity, a working group comprised of building science stakeholders was convened to advise this process, and to select an initial group of energy efficient wall assemblies to characterize that have a high-deployment potential, but are in need of a moisture risk assessment. Research will continue from FY15 to FY18. It is expected that wall designs for various US Climate Zones will begin to be issued in 2016. 


\section{BACKGROUND}

Energy-efficient walls with higher thermal insulation levels and tighter construction are being promoted by building codes and standards. This is demonstrated in the 2012 International Energy Conservation Code (IECC) and the Standard 90.1-2013 from the American Society of Heating, Refrigerating and AirConditioning Engineers (ASHRAE 2013a). However, the adoption rate of these and upcoming requirements by local governments will be highly dependent on being able to demonstrate to the construction industry that the long-term performance of walls is not negatively affected by the new energy efficient measures. In fact, some members of the construction industry have raised concerns about these requirements because they could lead to designs that are less forgiving, given that these walls are likely to have:

- Lower drying potential because of their higher insulation and airtightness levels.

- Materials with a lower water vapor permeance such as continuous foam insulation.

- Colder exterior sheathings during the winter when the increased levels of insulation are only provided in the wall cavity and exterior continuous insulation is not utilized. The side of the sheathing that is facing the wall cavity could then experience high relative humidity levels, and serve as a condensation surface and/or as an area that is prone to mold growth.

- Colder drywalls in exterior walls during the summer given the higher levels of cavity insulation. The drywall side that is facing the wall cavity could then experience high relative humidity levels, and serve as a condensation plane and/or as an area that could promote mold growth.

The goal of this multi-year project is to assess the moisture durability of energy-efficient walls in order to increase their adoption rate by supplying evidence to builders that these designs can be constructed in a manner that would avert potential future problems. A limited number of studies have aimed for a somewhat similar objective. However, these studies represent partial hygrothermal evaluations because they mostly relied on field tests (Fox 2014, Home Innovation Research Labs 2014), laboratory studies (Desmarais et al. 2000, Langmans et al. 2012), or simulations (Ojanen et al. 1994, Straube and Smegal 2012, Glass 2013, Lepage et al. 2013). In these evaluations, field and lab tests were limited to very specific scenarios, while results from simulations were not verified with empirical data. Moreover, moisture accumulation due to airflow was minimally accounted for even though researchers have raised concerns about the effects of airflow on the durability of building envelope assemblies (Ojanen et al. 1994, Desmarais et al. 2000, Künzel et al. 2011).

The present work aims to provide the residential construction industry with energy-efficient wall designs that are moisture durable based on a probabilistic risk assessment. The main differences between this evaluation and previous research are that the present study:

- Examines the effects of all sources of moisture in walls through a combination of simulations, lab experiments and field tests.

- Includes airflow among the sources of moisture because the effects of current airtightness requirements on moisture durability are not known and the effects of airflow have been primarily evaluated through simulation tools that have limited and unverified capabilities to address this phenomenon. 
- Utilizes a probabilistic approach that takes into account numerous variables, and therefore, expands the credibility, and thus the applicability of the results.

The end goal is to identify, in an accelerated and robust manner, wall assemblies that have the lowest probability of encountering moisture durability problems as illustrated in Figure 1.

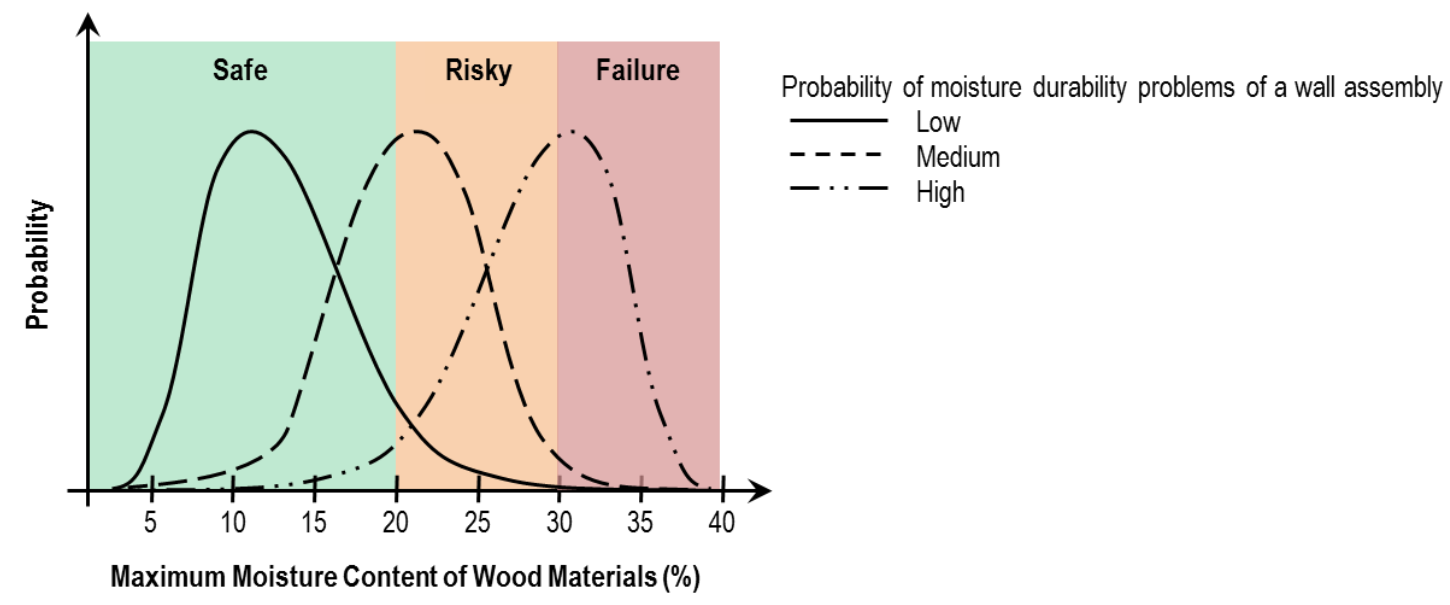

Figure 1. Example of probability density distribution of moisture durability problems in walls.

This report describes a proposed test protocol that will be followed in this multi-year project to identify energy-efficient and moisture durable wall assemblies. More specifically, this report covers the laboratory tests and simulations that can used to select energy-efficient wall assemblies that show a low probability of moisture durability problems, but require conclusive examinations through field evaluations. This approach is demonstrated with an example that illustrates the mathematical method that was followed to calibrate the selected simulation tool, type of laboratory tests that will be conducted to validate the calibrated model, and results from the probabilistic risk assessment using the calibrated simulation tool.

\section{PROPOSED TEST PROTOCOL}

The general approach of the proposed test protocol that will be used to evaluate the moisture durability of energy-efficient walls is described in Figure 2. In the first step, a wall assembly of interest is selected. The second step specifies how the wall assembly is expected to perform, and identifies the potential moisture durability risks and parameters that can affect these expectations. The next protocol step involves performing a quantitative hygrothermal assessment, which entails identifying the proper simulation tool(s) and determining whether the simulations will be based on deterministic or probabilistically varying input values. The selected simulation software then needs to be calibrated, or improved, because most of the tools available do not address airflow properly. Subsequently, laboratory and field measurements are used to validate the simulation software, and also to recalibrate it if necessary. After validation, the simulation tool will be used to evaluate the effect of the influential parameters in order to generate recommendations based on the expected hygrothermal performance. Although the combination of simulations, laboratory tests and field evaluations is typically ideal to obtain credible results, it may be possible to omit the laboratory and/or field tests. This will be the case when the calibrated simulation tool has already been validated for a wall assembly that is somewhat similar to the wall design in question, which will shorten the amount of time and decrease the cost to execute the proposed durability test protocol. 


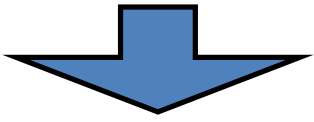

2. Identification of potential moisture durability problems

1. Expected hygrothermal performance and potential risks

2. Influential parameters

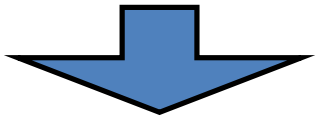

\section{Evaluation of moisture durability risks}

1. Simulations

1. Tools and modeling details

2. Calibration

3. Implementation of calibrated simulation tool

4. Analysis of results

2. Validation of simulations through laboratory and field tests

4. Recommendations based on expected hygrothermal performances

Figure 2. Proposed moisture durability test protocol.

\section{EXAMPLE OF IMPLEMENTATION OF TEST PROTOCOL}

\subsection{Selection of Wall Assembly}

There is a large selection of materials that can be incorporated into energy-efficient wall assemblies, although their properties and cost can vary substantially. Building America intends to use the durability test protocol that is described in this document to evaluate designs that have a high deployment potential. Input on this matter will be sought from experts in the residential construction sector with regard to cost, availability of materials, accessibility of trained personnel, and possible adoption rate by builders from different regions in the country. A similar approach is recommended to others who are planning to follow this protocol.

\subsection{Identification of Potential Moisture Durability Problems}

\subsubsection{Expected Hygrothermal Performance and Potential Risks}

It is expected that the structure of a house will not experience decay, drywall dissolution, corrosion of fasteners, and mold growth during its service life. Wood decay and corrosion can lead to structural failure (Merrill and TenWolde 1989), while mold growth can cause respiratory and asthma-related health problems (Fisk et al. 2007, Mendell et al. 2011). The present work follows the typical criteria to prevent 
decay fungi, which is to maintain the moisture content (MC) of wood below 20\% when temperatures range between 50 and $100^{\circ} \mathrm{F}$ (ASHRAE 2013b). Moreover, this research utilizes the mathematical model of mold growth developed by Hukka and Viitanen (1999) and its corresponding and updated mold index (Ojanen et al. 2010) to determine the likelihood of mold occurrence; this approach was selected because it is more thorough than ASHRAE Standard 160P (ASHRAE 2009). The mold index is described in Table 1 and is referenced throughout the results of this report.

Table 1. Mold growth index per Hukka and Viitanen (1999) and Ojanen et al. (2010).

\begin{tabular}{clc}
\hline Index & Description of Growth Rate & Risk Level \\
\hline 0 & No growth & Safe \\
\hline 1 & Small amounts of mold on surface (microscope), initial stages of local growth & Risky \\
\hline 2 & Several local mold growth colonies of surface (microscope) & Risky \\
\hline 3 & Visual findings of mold on surface, $<10 \%$ coverage, or $<50 \%$ coverage of mold (microscope) & Failure \\
\hline 4 & $\begin{array}{l}\text { Visual findings of mold on surface, } 10 \%-50 \% \text { coverage, or }>50 \% \text { coverage of mold } \\
\text { (microscope) }\end{array}$ & Failure \\
\hline 5 & Plenty of growth on surface, $>50 \%$ coverage (visual) & Failure \\
\hline 6 & Heavy and tight growth, coverage about $100 \%$ & Failure \\
\hline
\end{tabular}

\subsubsection{Influential Parameters}

There are numerous parameters that can affect the hygrothermal performance of energy-efficient wall assemblies. These parameters include:

1. Indoor environmental conditions (e.g., temperature, $\mathrm{RH}$, air pressure);

2. Outdoor environmental conditions (e.g., temperature, $\mathrm{RH}$, air pressure, solar radiation);

3. Hygrothermal material properties;

4. Material layout/geometry (i.e. design vs. actual construction);

5. Initial moisture content of materials;

6. Radiational properties of exterior façade; and

7. Airflow through the wall assembly (e.g., path, flow rate, direction)

Among these parameters, the hygrothermal effect of airflow through the wall assembly is the least understood and characterized in hygrothermal modeling and simulation tools. The main reason behind this knowledge gap is that simulation tools that attempt to evaluate the effect of airflow (e.g., WUFI ${ }^{\circledR} 1 \mathrm{D}$, WUFI $^{\circledR} 2 \mathrm{D}$ and Transient-Coupled-Conduction and Convection in 2D) have not been calibrated and/or validated. Moreover, although Desmarais et al. (2000), Langmans et al. (2012) and Fox (2014) have gathered laboratory or field data, none of them have used it to validate simulation tools that predict hygrothermal performance. This is particularly problematic because the quantitative assessment performed as the next step in this protocol relies on the effective simulation and characterization of the impact of the influential parameters on the moisture content of the wall assembly. Consequently, the following questions regarding airflow must be further assessed before proceeding:

- Is the influence of airflow on the moisture durability of the wall assembly significant, such that it must be included in the hygrothermal risk assessment?

- If air is determined to be a significant influential parameter, how can the selected hygrothermal modeling and simulation tool be augmented to adequately characterize the impact of airflow in combination with the other influential parameters? 


\subsubsection{Significance of Air Leakage on Moisture Durability}

Air leakage from both the indoor and outdoor environment can affect the moisture durability of building assemblies. The direction and magnitude of airflow through building components depend on variables such as pressure gradients (induced by wind loads, buoyant forces, and HVAC/ventilation systems), and the air permeability of the building materials. These airflows will result in an energy penalty due to air traveling to and/or from a conditioned space. However, there is a moisture durability aspect to air leakage that needs to be addressed as well. If the dew-point temperature of the air is higher than or similar to the material surface temperatures along the air leakage path, a high relative humidity (RH) or condensation may occur on these surfaces. These two conditions are essential for mold growth, corrosion of fastener, dissolution of drywall, and rot in wood.

Warm, moist air can transfer both moisture and heat to the surrounding materials in an air leakage path. It might seem that the transferred heat would raise the temperature enough to inhibit condensation; however, this phenomenon is more complicated as explained in the text that follows.

Figure 3 shows why condensation may occur on material surfaces within an air leakage path by illustrating two arbitrary hygrothermal conditions in a psychometric chart. The red dot on the bottom left corner of Figure 3 indicates an arbitrary condition within the air leakage path with a relatively low temperature, low relative humidity, and no airflow. The second case, which is illustrated by the red dot on the top right corner, represents the hygrothermal conditions of the environment from which the inflowing air originates, i.e., the source. Once inflow takes place, the steady-state values reached by the air within the path will be a function of the hygrothermal properties and conditions of the surrounding materials, the conditions of the source, and the airflow rate. For most building materials, under a constant flow rate, steady-state values will come closer to the incoming air's moisture conditions than to its temperature. This phenomenon is due to the heat and moisture transfer and storage properties of materials. In typical wood-based materials, heat is transported more efficiently than moisture. Consequently, any heat that the inflowing air may bring will be distributed amongst adjacent materials quickly, while the moisture in the incoming air will be transported through the wood at a relatively slow pace. Therefore, the temperature of the air that travels through the air leakage path is quickly influenced by the temperature of the surrounding materials, while the moisture levels of the air remain close to those of the environment from which the air originates. With relatively high flow rates, the temperature of the air will move closer to that of the incoming air. This is demonstrated by the dash lines in Figure 3, which illustrate the theoretical direction in which the hygrothermal conditions, of the air inside the leakage path, travel as they reach steady state. 


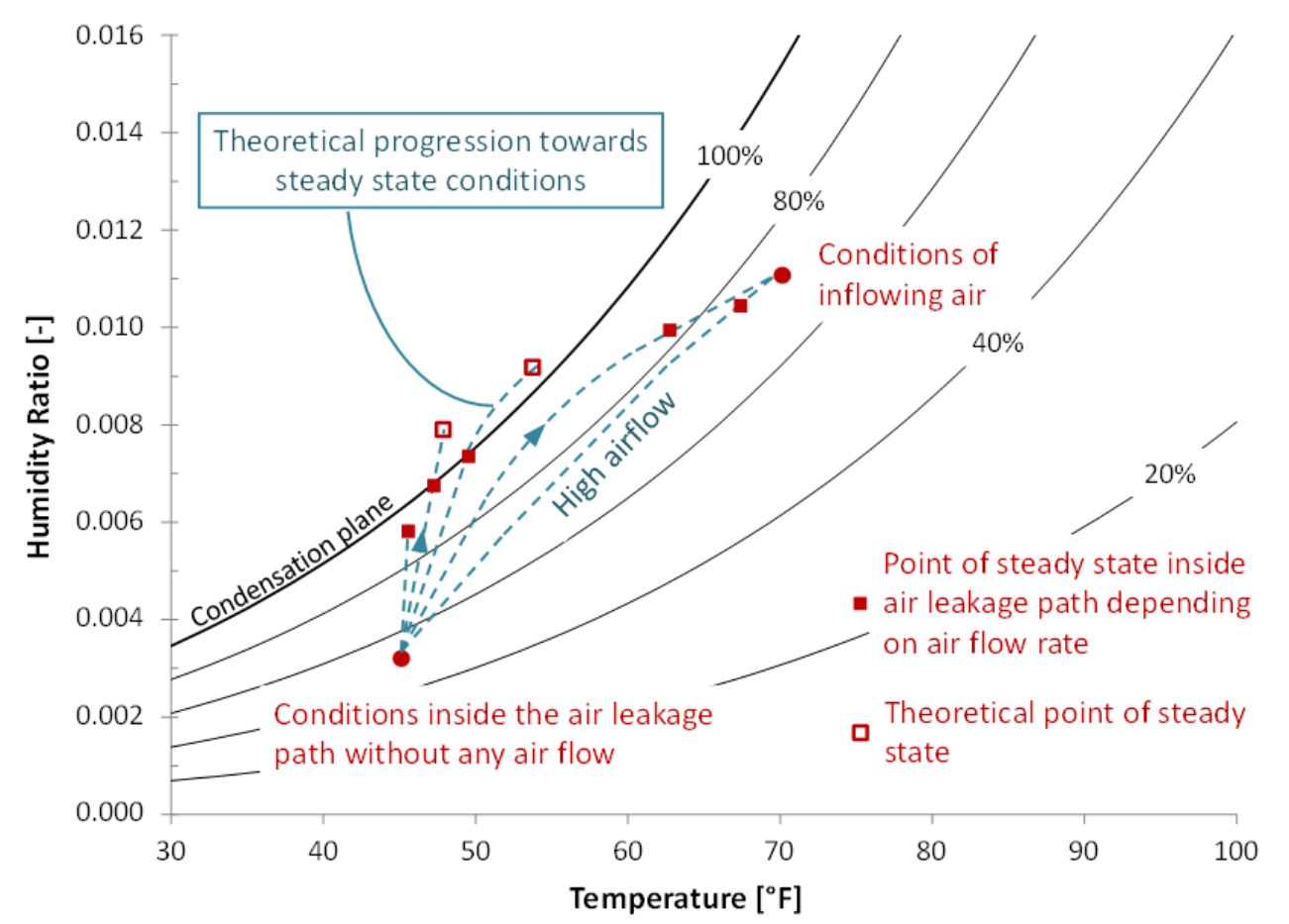

Figure 3. Theoretical steady-state hygrothermal conditions of air inside a leakage path at different flow rates.

The exchange of heat and moisture between the surfaces surrounding the air leakage path and the inflowing air will also depend on the distance between the air's entry point and the evaluation point. This is because heat and moisture are continuously exchanged between the air and its surrounding materials as the air moves along the flow path. This is illustrated in Figure 4, which shows how a step change in moisture and temperature, due to air leakage, will affect the conditions along an air leakage path. In this figure, (0) represents the initial hygrothermal conditions of the air within the leakage path before airflow occurs, and (1) represents the conditions of the inflowing air. The three distances are with respect to the air's entry point. As seen in the figure, moisture conditions are similar regardless of the location along the leakage path. The significance of these theoretical results is that the humidity ratio of the air at any point within the flow path will be closely coupled to that of the incoming air at relatively low flow rates, while the temperature of the air within the flow path will vary throughout the flow path. Therefore, high relative humidity levels may occur on the subsequently colder surfaces within the air leakage path, since the relative humidity is a function of both temperature and moisture levels. 


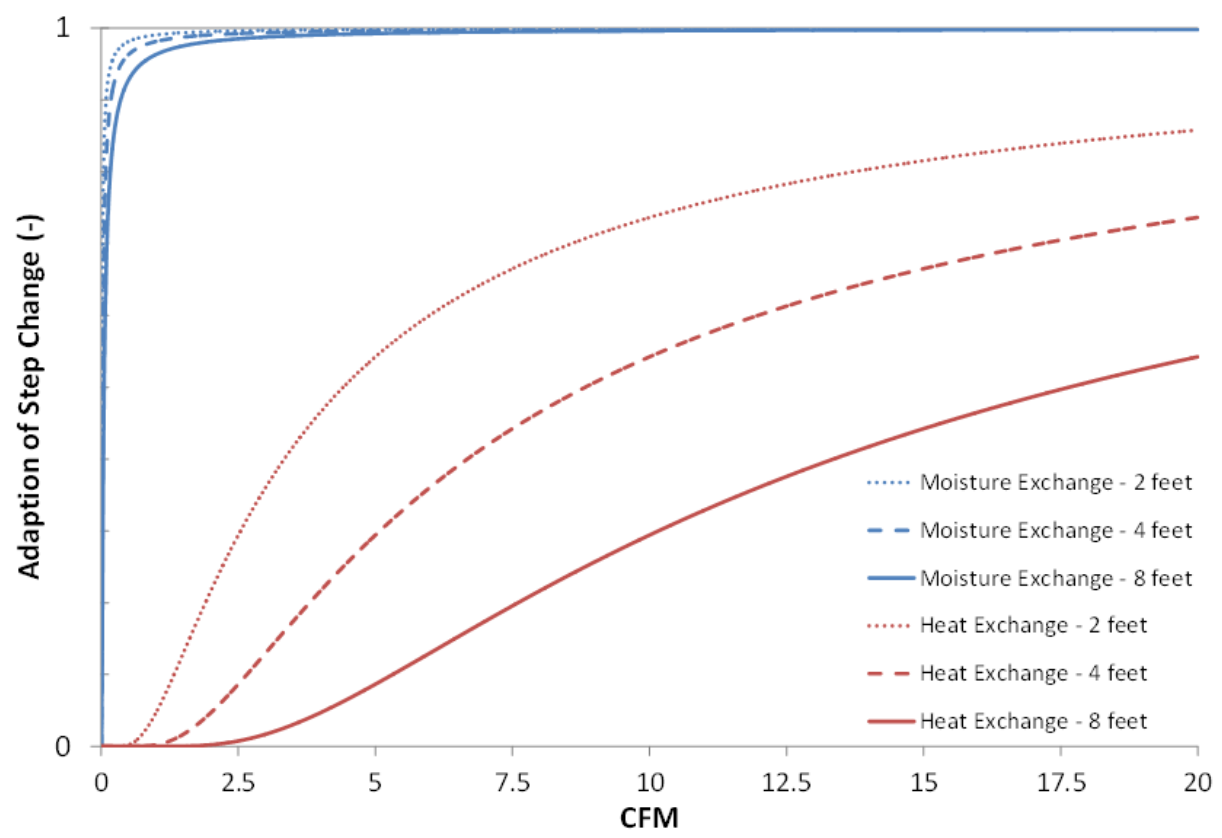

Figure 4. Steady-state hygrothermal conditions along an air leakage path after a step change in flow rate.

As described, air leakage is in fact a significant influencing parameter on moisture durability, and thereby should be included in hygrothermal simulations. Therefore, one of the primary goals of this multi-year project will be to determine the critical airflow rate $\left(Q_{c r}\right)$ for a wall assembly under certain indoor and outdoor conditions. Figure 5 describes the effect of various exfiltration rates in a wall that is located in a cold climate. As illustrated in Figure 5, $Q_{c r}$ is the lower boundary of the critical airflow range, and flows lower than $Q_{c r}$ have a low probability of resulting in durability problems. In contrast, air leakage rates within the critical airflow range are likely to accumulate moisture that can compromise the long-term performance of the wall. Rates that are higher than the critical airflow range may be capable of removing significant amounts of moisture from the wall cavity during the summer and shoulder months when indoor moisture loads tend to decrease. It is important to note that this relationship between moisture accumulation and airflow rate is highly dependent on the environmental conditions and material properties. 


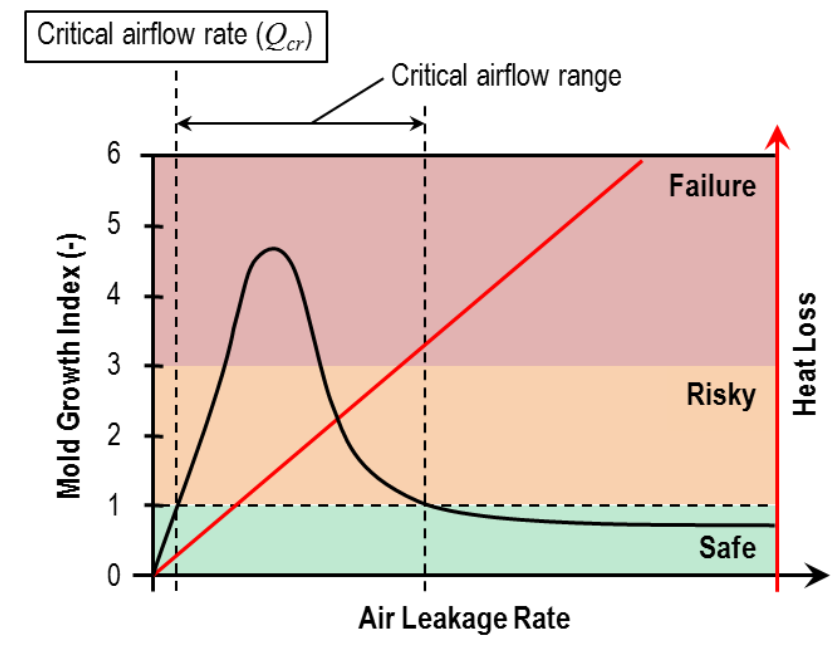

Figure 5. Mold growth index as a function of exfiltration rates in a cold climate.

\subsection{Evaluation of Moisture Durability Risks}

The next step in the hygrothermal risk assessment is to perform a quantitative analysis, where multiple scenarios will be evaluated through simulations. Preferably, the simulations are based on stochastically varying input parameters that will generate results that can be evaluated in a probabilistic manner. A limited number of parameters were varied in the present implementation of the proposed test protocol because the objective was to provide an example of the type of results that the protocol will supply. The full implementation of this comprehensive stochastic assessment will take into account all of the previously mentioned influential parameters and their probabilistic distributions to supply designs of energy-efficient wall assemblies for various climate zones.

\subsubsection{Simulations}

Simulations are usually a cost and time-efficient approach to evaluate the hygrothermal performance of a building component. However, the available calculation tools are not complete and fully validated to account for the influence of airflow on hygrothermal performance. This section presents an approach to calibrate simulations through a mathematical method that improves the procedure in which the effect of air leakage on building components is modeled. Thereafter, results are validated with laboratory measurements.

\subsubsection{Tools and Modeling Details}

There are several hygrothermal tools available to mimic transient heat, air and moisture transfer in building components. WUFI ${ }^{\circledR}$ (Künzel 1995) was selected for this study since it has been frequently validated against experimental data (Kehrer and Schmidt 2008). Unfortunately, WUFI ${ }^{\circledR}$ has a simplified method for dealing with airflow that doesn't comply with true conditions (Künzel et al. 2011). For this reason, the $\eta$-method has been developed to allow WUFI $^{\circledR}$ to handle air leakages differently, as described in Section 3.3.1.3.2. As of today, there are no hygrothermal tools that are readily available and that can conduct multiple probabilistic simulations. However, Holm (2001) and Pallin and Kehrer (2013) were able to automatically run multiple simulation scenarios with WUFI ${ }^{\circledR} 1 \mathrm{D}$ by making adjustments to the calculation code. Because air leakages are seldom a 1-D phenomenon, part of this study involves enabling WUFI ${ }^{\circledR} 2 \mathrm{D}$ to perform multiple simulation runs. 


\subsubsection{Calibration of the Hygrothermal Model for Air Leakage - The $\eta$-Method}

The steady state 1-D thermal conditions of any location inside a building component can be described through a network analysis as shown below.

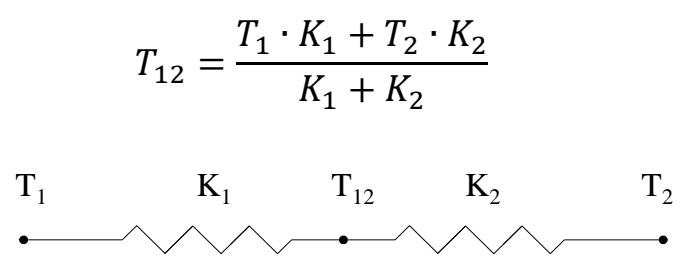

Here, $T$ is the temperature $\left({ }^{\circ} \mathrm{F}\right)$ and $K$ is the material thermal conductance $\left(\mathrm{Btu} /\left(\mathrm{hr} \cdot{ }^{\circ} \mathrm{F}\right)\right) . T_{1}, T_{2}$ and $T_{12}$ are the temperatures of the outdoor air, indoor air, and air within the leakage path, respectively; while $K_{l}$ and $K_{2}$ are the thermal conductance of the materials between these points. In a steady state 1-D thermal condition, air leakage can be included into Eq. (1) as a heat source or sink, $I$ (Btu/hr), as indicated below.

$$
T_{12}=\frac{\left(T_{1}+\frac{I}{K_{1}}\right) \cdot K_{1}+T_{2} \cdot K_{2}}{K_{1}+K_{2}}
$$

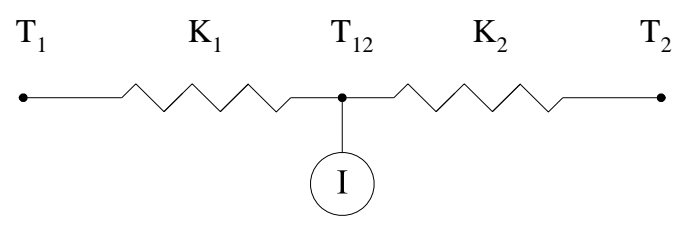

where $I$ is defined as,

$$
I=\rho_{\text {air }} \cdot c_{\text {air }} \cdot\left(T_{2}-T_{12}\right) \cdot Q
$$

Here, $\rho\left(\mathrm{lb} / \mathrm{ft}^{3}\right)$ and $c\left(\mathrm{Btu} /\left(\mathrm{lb} \cdot{ }^{\circ} \mathrm{F}\right)\right)$ are the density and specific heat capacity of the air, respectively, and $Q$ $\left(\mathrm{cfm}\right.$ or $\left.\left.\mathrm{ft}^{3} / \mathrm{s}\right)\right)$ is the air leakage rate.

Air leakage is simulated in $\mathrm{WUFI}^{\circledR} 1 \mathrm{D}$ and $\mathrm{WUFI}^{\circledR} 2 \mathrm{D}$ as an air change rate $(\mathrm{ACH})$ that originates from either the indoor or the outdoor environment, and is distributed uniformly over a defined length in onedimensional cases or an area in two-dimensional cases. This approach complies with the method described in Eq. (2) and can be expected to estimate 1D heat and moisture transfer accurately. For 2D transfer, this approach is questionable since the exchange of heat and moisture within an air leakage path is dependent on the path length as illustrated in Figure 4. Therefore, a more appropriate 2D solution is considered in this work:

$$
T_{12}(x)=T_{1}+\left(T_{2}-T_{1}\right) \cdot e^{\left(\frac{-x}{l_{c}}\right)}
$$

where $x(\mathrm{ft})$ is the distance between the entry point of the air into the air leakage path and the point of evaluation, and $l_{c}(\mathrm{ft})$ is the characteristic length per

$$
l_{c}=\frac{\rho_{\text {air }} \cdot c_{\text {air }} \cdot Q}{L_{\text {surf }} \cdot K}
$$


Here, $L_{\text {surf }}(\mathrm{ft})$ is the surface length or the perimeter of the cross section through the air leakage path, and $K$ is the effective thermal conductance of the materials surrounding the air leakage path.

Figure 5 compares the solutions from Eq. (2) and (4), and indicates a large discrepancy between their results. The thermal conductances and geometrical characteristics used in these estimates belong to those from the test specimen described in Section 4.3.1.3.2. Since Eq. (4) is dependent on the distance that the air has traveled within the leakage path, solutions are presented at five different locations. A seen in Figure 5, when air flows at a rate of $5 \mathrm{cfm}, \mathrm{Eq}(2)$ predicts a constant air temperature of $57^{\circ} \mathrm{F}$ along the airflow path. In contrast, Eq (4) indicates that temperatures will vary from $51^{\circ} \mathrm{F}$ to $64^{\circ} \mathrm{F}$ at distances that range from one to eight feet from the air's entry point.

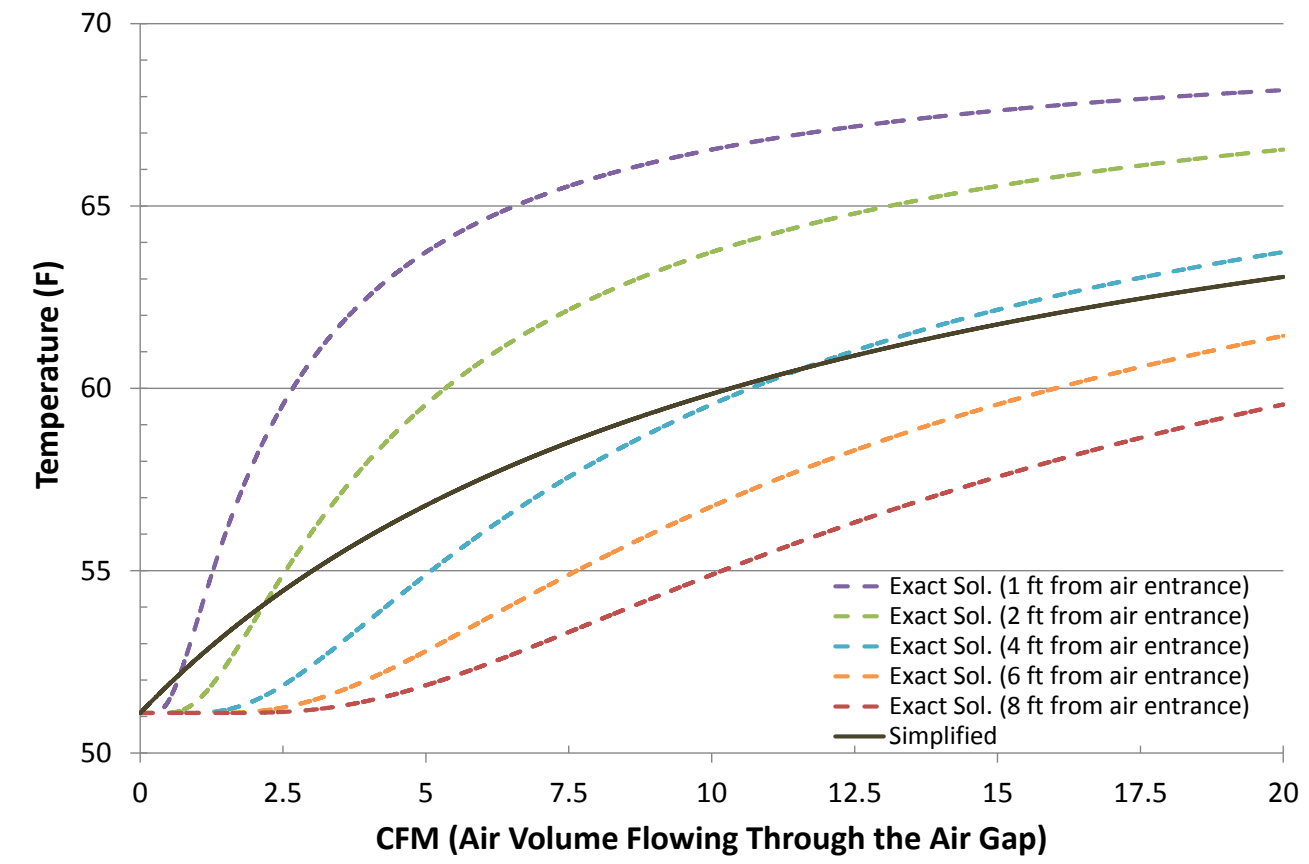

Figure 6. Calculated vs. simplified steady-state solutions for heat exchange in an air channel.

As has been previously discussed, WUFI ${ }^{\circledR} 1 \mathrm{D}$ and $\mathrm{WUFI}^{\circledR} 2 \mathrm{D}$ distribute the air leakage uniformly over a defined path or area. Since Eq. (2) is not valid for 2D air leakage paths, the suggested approach is to create a correction factor, $\eta$, which allows the solution of Eq. (2) to match that of Eq. (4). This correction factor can then be incorporated into $\mathrm{WUFI}^{\circledR}$ through an air changes per hour $(\mathrm{ACH})$, which is derived from the adjusted source/sink, $I_{\eta}$, expressed as:

$$
I_{\eta}=\eta(x, Q) \cdot I
$$

where, $\eta$ is a function of both the distance into the air leakage path and the air flow rate.

If the solution of Eq. (2) includes $I_{\eta}$ instead of $I$, the following can be stated:

$$
\begin{aligned}
& T_{12}(x)=T_{12} \\
& T_{12}(x)=\frac{\left(T_{1}+\frac{I_{\eta}}{K_{1}}\right) \cdot K_{1}+T_{2} \cdot K_{2}}{K_{1}+K_{2}}
\end{aligned}
$$


$I_{\eta}$ can be expressed as:

$$
I_{\eta}=\left(\frac{T_{12}(x) \cdot\left(K_{1}+K_{2}\right)-T_{2} \cdot K_{2}}{K_{1}}-T_{1}\right) \cdot K_{1}
$$

Finally, $\eta$ can be derived using Eq. (3), (6) and (9) resulting in

$$
\eta(x, Q)=\frac{\left(\frac{T_{12}(x) \cdot\left(K_{1}+K_{2}\right)-T_{2} \cdot K_{2}}{K_{1}}-T_{1}\right) \cdot K_{1}}{\rho_{\text {air }} \cdot c_{\text {air }} \cdot\left(T_{2}-T_{12}\right) \cdot Q}
$$

In accordance with Eq. (3) and (6), $I_{\eta}$ can be expressed as a function of $\mathrm{ACH}$, which can be used as an input for air leakage in WUFI ${ }^{\circledR}$.

$$
\begin{aligned}
& I_{\eta}=\eta(x, Q) \cdot A C H \cdot \frac{V}{3600} \cdot \rho_{\text {air }} \cdot c_{\text {air }} \cdot\left(T_{2}-T_{12}\right) \\
& A C H_{\eta}=\eta(x, Q) \cdot A C H
\end{aligned}
$$

where $Q\left(\mathrm{ft}^{3} / \mathrm{s}\right)$ is expressed as $A C H \cdot \frac{V}{3600} \cdot \mathrm{ACH}_{\eta}$ is the corrected air change rate that is used as an input in $\mathrm{WUFI}^{\circledR}$.

In the present work, $\eta$ was calculated only as a function of $x$ and $Q$ because preliminary analyses indicated that changes in $T_{1}, T_{2}, K_{1}$ and $K_{2}$ have minimal effects on this correction factor. This assumption will be examined further in future evaluations.

An analogous procedure can be followed with moisture transfer (Hagentoft 2001) as seen in Eq. (4)

$$
v_{12}(x)=v_{1}+\left(v_{2}-v_{1}\right) \cdot e^{\left(\frac{-x}{l_{c_{v}}}\right)}
$$

where $v\left(\mathrm{lb} / \mathrm{ft}^{3}\right)$ is the moisture content of the air along the leakage path, $l_{c_{v}}(\mathrm{ft})$ is the characteristic length for moisture transfer, and $K_{v}(\mathrm{ft} / \mathrm{s})$ is the water vapor transport conductance.

$$
l_{c_{v}}=\frac{Q}{L_{\text {surf }} \cdot K_{v}}
$$

Eq. (12) and (13) can be used in conjunction with the procedure described above to estimate moisturerelated correction factors and sources. However, as seen in Figure 4, the length of the path has a greater effect on heat exchange than on moisture exchange. Therefore, efforts were focused on generating correction factors for the temperature analyses rather than the moisture analyses.

\subsubsection{Initial Validation of Calibrated Simulation Tool}

Laboratory tests were conducted to validate the mathematically-derived correction factors. The general approach involved creating a defined airflow path in a test wall, regulating the flow rate, and monitoring the temperature and relative humidity of the air along the flow path. These measurements were then used to conduct initial validations of the results. Details of this approach are described hereafter. 


\subsection{Heat, Air and Moisture Penetration Chamber}

Experiments were conducted in a heat, air and moisture (HAM) penetration chamber shown in Figure 7. The chamber is composed of an indoor room (IR), an outdoor room (OR) and a test frame, all of which are thermally isolated from the lab space. The indoor room and the test frames are movable so test walls that are up to $8^{\prime}-0$ " wide by $10^{\prime}-0$ " tall can be built outside the chamber. Table 2 lists the parameter ranges that the chamber is capable of monitoring with the sensors that are described in Table 3 . Most of these parameters can be regulated with proportional-integral-derivative controllers (Yokogawa UT55A) and LabVIEW 11.0.1. Patch panels within the IR and OR allow for sensors to be installed on both sides of the air and water-resistive barriers without making penetrations through them.

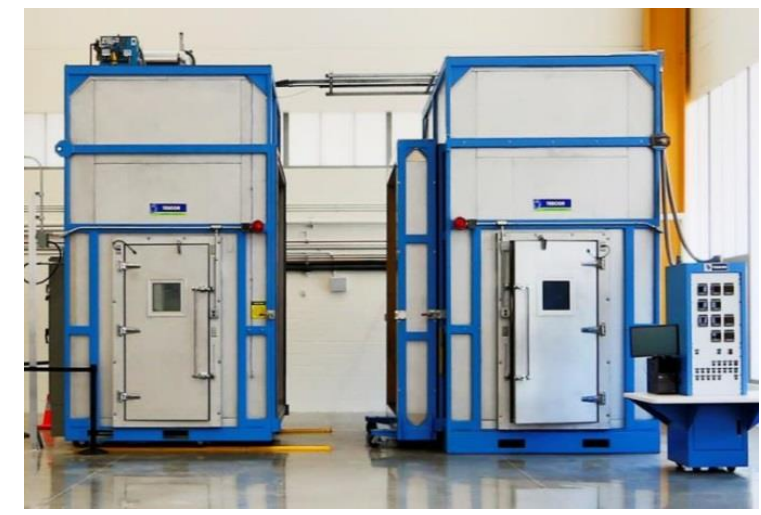

Figure 7. Heat, air and moisture penetration chamber.

Table 2. Parameters controlled in the heat, air and moisture penetration chamber

\begin{tabular}{lcc}
\hline Parameter & Indoor Room & Outdoor Room \\
\hline Temperature $\left({ }^{\circ} \mathrm{F}\right)$ & 60 to 90 & 0 to 110 \\
\hline Relative humidity $(\%)$ & 10 to 90 & 10 to 90 \\
\hline Pressure $(\mathrm{psf}, \mathrm{Pa})$ & $\pm 1.57, \pm 75$ & $\pm 31.3, \pm 1500$ \\
\hline Infrared radiation $\left(\mathrm{W} / \mathrm{ft}^{2}\right)$ & $\mathrm{NA}$ & $\leq 100$ \\
\hline Rain water flow rate $(\mathrm{ft} 2 / \mathrm{min})$ & $\mathrm{NA}$ & $\leq 1$ \\
\hline Rain water temperature $\left({ }^{\circ} \mathrm{F}\right)$ & $\mathrm{NA}$ & 40 to 95 \\
\hline
\end{tabular}

Table 3. Sensors.

\begin{tabular}{llcl}
\hline Sensor & Model & Range & Accuracy \\
\hline Indoor room & & & \\
\hline Temperature & Vaisala HMT 330 & -40 to 176 & $\pm 0.36^{\circ} \mathrm{F}$ at $68^{\circ} \mathrm{F}$ \\
\hline Relative humidity (\%) & Vaisala HUMICAP 180 & 0 to 97 & $\begin{array}{l} \pm 0.6 \% \mathrm{RH}(0 \text { to } 40 \% \mathrm{RH}) \text { at } 68^{\circ} \mathrm{F} \\
\pm 1 \% \mathrm{RH}(40 \text { to } 97 \% \mathrm{RH}) \text { at } 68^{\circ} \mathrm{F}\end{array}$ \\
\hline Pressure (psf) & Rosemount 2051CD & $\pm 288,000$ & $\pm 0.075 \% \mathrm{FS}$ \\
\hline Outdoor room & & & \\
\hline Temperature $\left({ }^{\circ} \mathrm{F}\right)$ & Vaisala HMT 330 & -40 to 176 & $\pm 0.36^{\circ} \mathrm{F}$ at $68^{\circ} \mathrm{F}$ \\
\hline Relative humidity (\%) & Vaisala HUMICAP 180 & 0 to 97 & $\pm 0.6 \% \mathrm{RH}(0$ to $40 \% \mathrm{RH})$ at $68^{\circ} \mathrm{F}$ \\
\hline Pressure (psf) & Rosemount 2051CD & $\pm 288,000$ & $\pm 0.075 \% \mathrm{RH}(40$ to $97 \% \mathrm{RH})$ at $68^{\circ} \mathrm{F}$ \\
\hline
\end{tabular}




\begin{tabular}{|c|c|c|c|}
\hline \multicolumn{4}{|l|}{ Test wall } \\
\hline Temperature $\left({ }^{\circ} \mathrm{F}\right)$ & $\begin{array}{lrrr}\text { No } 26 \text { AWG } & \text { Type } & \text { T } \\
\text { copper/constantan } & & \\
\text { thermocouples } & & \end{array}$ & & Deviates $-2^{\circ} \mathrm{F}$ at $212^{\circ} \mathrm{F}$ \\
\hline Relative humidity (\%) & Honeywell HIH-4000-003 & 0 to $100 \%$ & $\pm 3.5 \% \mathrm{RH}$ \\
\hline \multicolumn{4}{|l|}{ Miscellaneous } \\
\hline Airflow meter (SCFM)* & EPI 8732 MPNH-SSS-133-DC24 & 0.16 to 32 & $\pm\left[1 \% \mathrm{RDG}+\left(0.5 \% \mathrm{FS}+0.02 \% \mathrm{FS} /{ }^{\circ} \mathrm{C}\right)\right]$ \\
\hline
\end{tabular}

${ }^{*}$ Flow measurements are standardized to $70^{\circ} \mathrm{F}$ and 29.92 in. of mercury.

\subsection{Test Setup}

The wall assembly selected to implement the proposed moisture durability test protocol had materials that comply with the 2012 International Residential Code (IRC) in climate zones 1 through 5. It consisted of vinyl siding, a mechanically-fastened water-resistive barrier, 7/16" oriented strand board (OSB), $2 \times 6$ studs at 24" on center, R19 kraft-faced fiberglass insulation, and drywall.

There are numerous paths that air could follow in a wall assembly as shown in Figure 8, but their effect on moisture durability is dependent on the previously mentioned influential parameters. Ojanen et al. 1994 and Desmarais et al. (2000) examined the effect of a few airflow paths through simulations and lab experiments, respectively. Their results indicate that indirect paths where air has more contact with susceptible materials are more detrimental to moisture accumulation than direct paths where air flows straight through the wall cavity. Based on these results, the present work explored the path that Ojanen et al., and Desmarais et al. associated with moisture problems: exfiltration in a cold climate where air enters the wall cavity at the top of the wall, travels alongside the OSB, and exits at the bottom of the wall. Figure 9 describes the material layout in the test wall and the previously mentioned airflow path, as well as how air exfiltrates in an actual wall. Two cavities were included in the test wall: Wall Cavity A (WCA) and Wall Cavity B (WC-B). The difference between these two is that WC-B served as a control given that the sides of the wood members facing the cavity were coated with oil-based polyurethane to reduce the effect of sorption onto these surfaces. The test wall did not include the vinyl siding and the mechanically-fastened water-resistive barrier in order to limit the number of penetrations in the OSB that could affect the studied airflow path. The lack of these two materials should not affect the results because their thermal and water vapor resistance is minimal.

Exfiltration

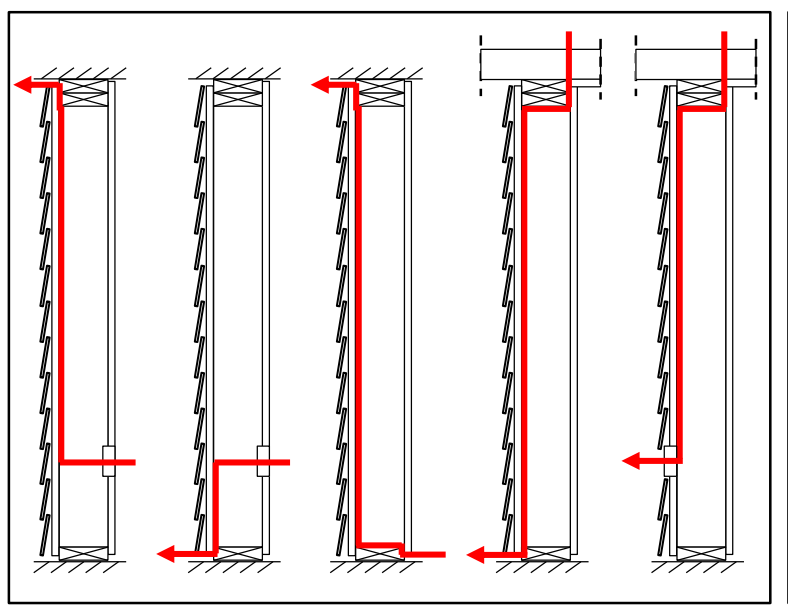

Infiltration

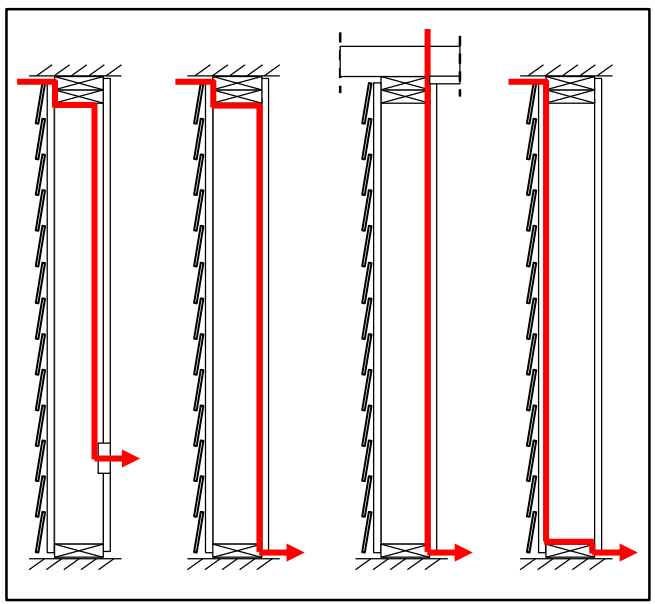

Figure 8. Potential exfiltration and infiltration airflow paths through exterior walls. 
As shown in Figure 10, the airflow path was incorporated into the test wall with a netting made of polypropylene twine that would maintain a somewhat uniform $1 / 2$ " gap between the fiberglass batts and the OSB. This airflow path was connected to the IR by a $1 / 2$ " gap between the fiberglass insulation and the top plate that continued through the drywall, and to the OR by a 1/16" gap between the OSB and the bottom plate. These were the two primary points of airflow through the test wall given that special attention was taken to seal most of the other potential leakage spots with caulk or tape. The test standard ASTM E283 (ASTM 2004) was followed to determine the airflow equations for WC-A and WC-B:

$$
\begin{array}{ll}
Q_{W C-A}=9.73 \Delta P^{0.62} & \text { where } Q \text { is in cfm and } \Delta \mathrm{P} \text { is in psf } \\
Q_{W C-A}=0.42 \Delta P^{0.62} & \text { where } Q \text { is in } \mathrm{L} / \mathrm{s} \text { and } \Delta \mathrm{P} \text { is in Pascal } \\
Q_{W C-B}=10.2 \Delta P^{0.65} & \text { where } Q \text { is in cfm and } \Delta \mathrm{P} \text { is in psf } \\
Q_{W C-B}=0.39 \Delta P^{0.65} & \text { where } Q \text { is in } \mathrm{L} / \mathrm{s} \text { and } \Delta \mathrm{P} \text { is in Pascal }
\end{array}
$$

Flow rates per these equations were intended to be relatively high in order to reduce uncertainties from the measured data.

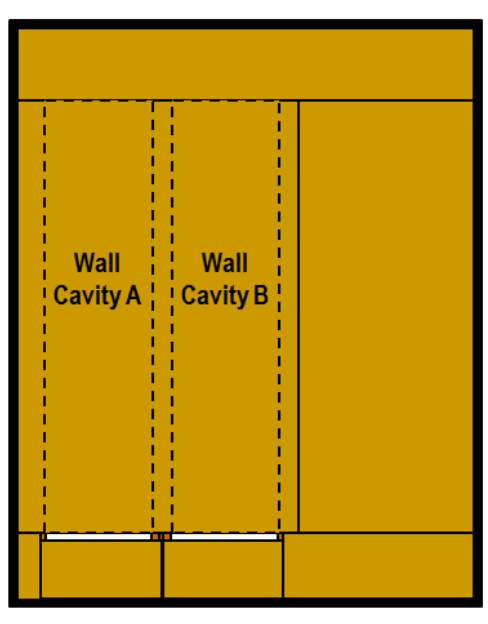

Elevation of test wall facing outdoor room
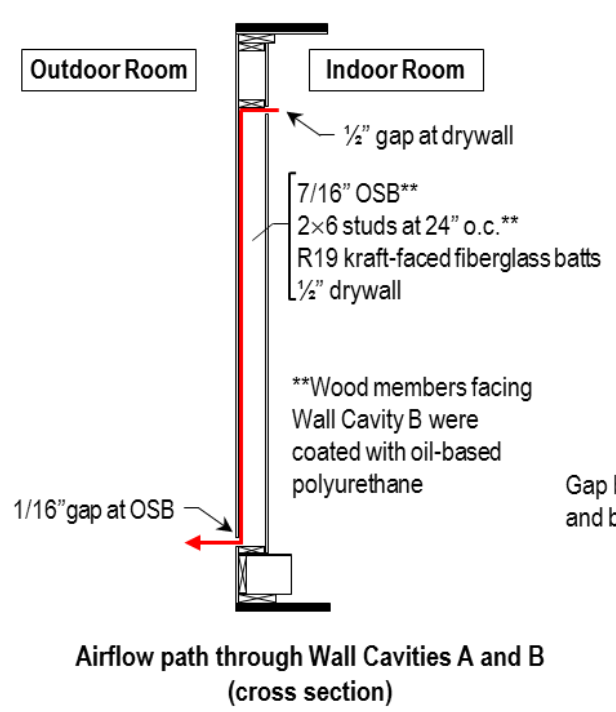

Gap between OSB and bottom plate

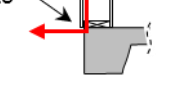

Actual airflow path

Figure 9. Material assembly in test wall, and evaluated and actual airflow path.

Table 3 lists the temperature and relative humidity $(\mathrm{RH})$ sensors that were used to instrument the test wall. These sensors were calibrated within the HAM chamber while connected to the data acquisition system. Relative humidity sensors were paired with thermocouples and wrapped with a 58-perm membrane to protect the RH sensors from water. Figure 11 shows the sensor layout within the wall cavity and on the exterior side of the OSB. Temperature and RH sensors throughout the airflow path permitted estimating the humidity ratio and water vapor content in the air as it exfiltrated the wall, which is equivalent to being able to monitor the moisture source along the leakage path.

In the present work, winter conditions were simulated with indoor temperature and $\mathrm{RH}$ set to $70^{\circ} \mathrm{F}$ and $50 \%$, respectively. Similarly, outdoor conditions were $50^{\circ} \mathrm{F}$ and $30 \%$. These values were selected in part to prevent condensation within the air gap. The test wall was subjected to these temperature and $\mathrm{RH}$ values, and minimal flow through the air gap (i.e., $\Delta \mathrm{P} \cong 0$ ) for about 24 hours before the test was initiated. Exfiltration was achieved by maintaining the indoor room at a higher pressure than the outdoor room. The effect of different airflow rates was explored by varying the pressure difference across the wall every 
two hours per the following sequence: $0.1,0.21,0.42,0.84,0.42,0.21,0.1$ psf $(5,10,20,40,20,10,5$ $\mathrm{Pa}$ ). These step changes in pressure and flow rate are illustrated in Figure 12. During the tests, data were collected every 10 seconds, and one-minute averages are reported in this document.

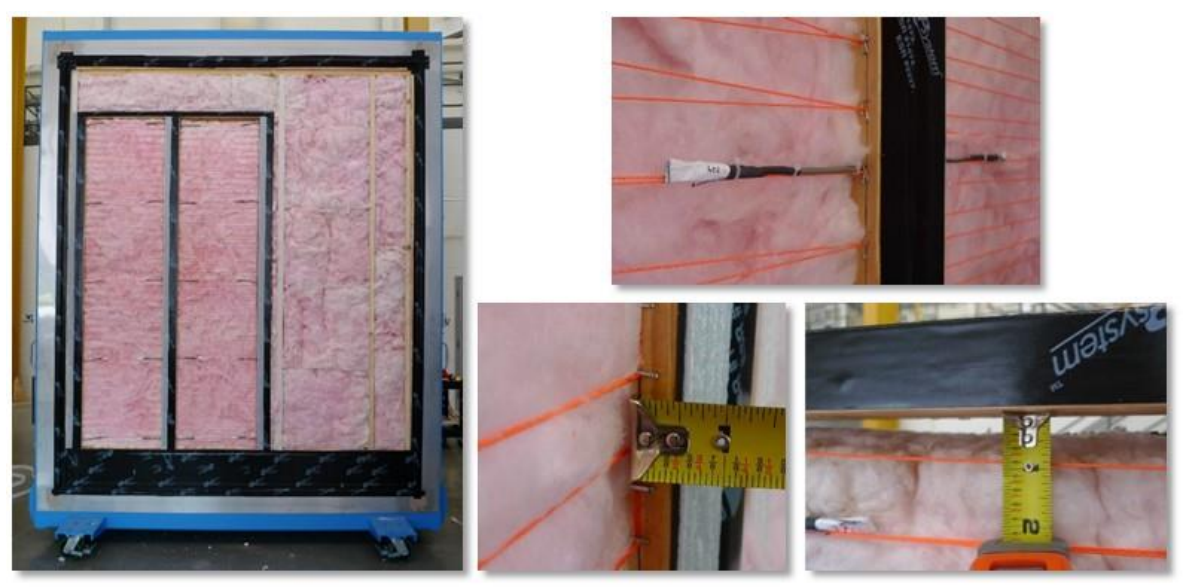

Figure 10. Airflow path between fiberglass insulation and $\mathrm{OSB}$, and insulation and top plate.

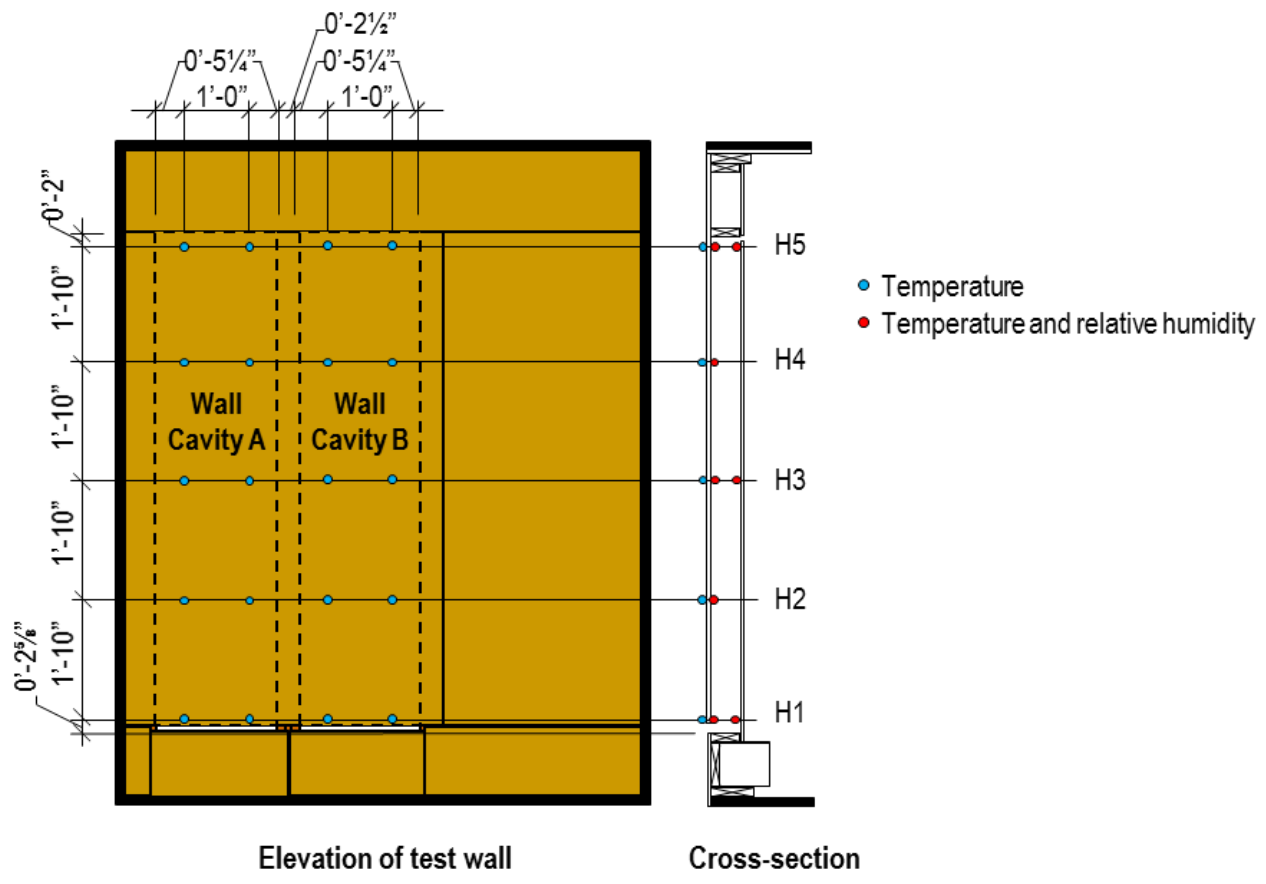

Figure 11. Sensor layout in test wall. 

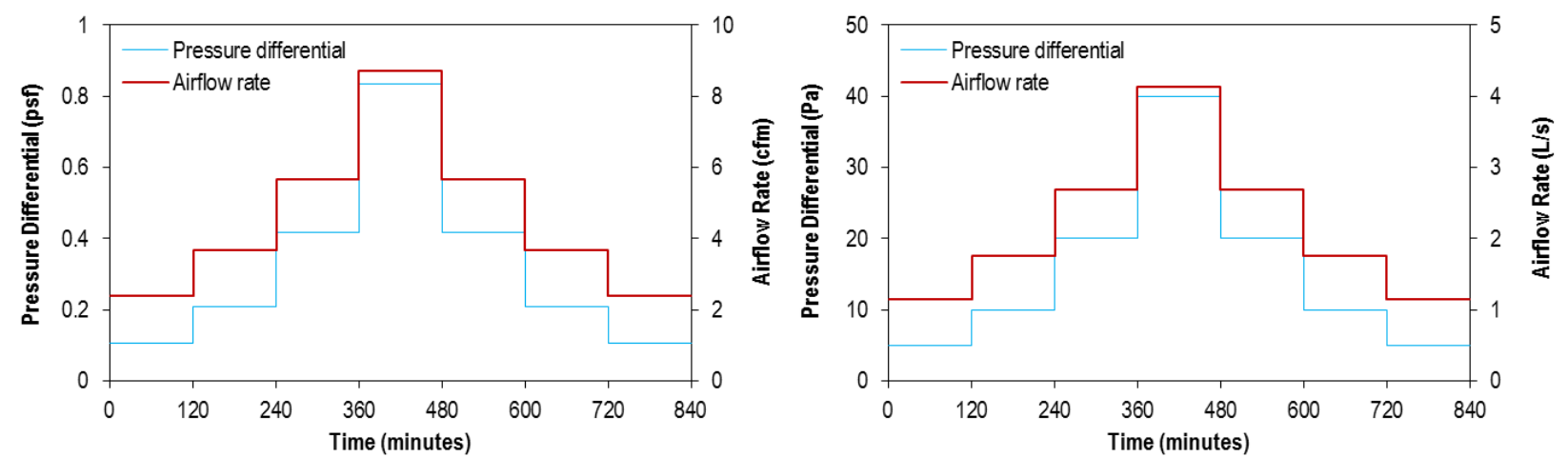

Figure 12. Followed sequence of indoor-to-outdoor pressure differentials across the test wall.

The main difference between the test setup just described and the experiments conducted by others is that one of the key goals of the present work is to reduce the amount of time it takes to run a test. The evaluations conducted or proposed by Desmarais et al. (2000), Cornick (2008), and Langmans et al. (2012) sought for the moisture content in the building materials to reach steady-state or quasi-steadystate, which involved weeks or months. On the contrary, the present research focuses on using laboratory measurements to study moisture sources within the wall, which in this case is the airflow through the wall cavity. The materials in the wall cavity will respond accordingly to this moisture source; this process will be accounted for through simulations to expedite hygrothermal evaluations.

\subsection{Collected Data}

Table 4 and Figure 13 describe the conditions in the indoor and outdoor rooms during the test. Most of the measurements were close to the set points with two exceptions. The average indoor-to-outdoor pressure differential was typically about $0.02 \mathrm{psf}(1 \mathrm{~Pa})$ greater than the set point as illustrated in Table 4 and Figure 14, where the latter also includes the calculated flow rates based on Equations 14 through 17. Moreover, the relative humidity in the OR was greater than $30 \%$ during most of the test. The reason behind this is that the OR has two mechanisms to dehumidify: cooling and supplying dry air into the room; and it is likely that minimal cooling was needed and that the dry air flow rate was not sufficient to offset the moist air exfiltrating from the IR into the OR. Nevertheless, the relative humidity in the OR had minimal effects on the experiment given that the air was flowing from the IR into the OR, and because the indoor room served as the leading source of moisture within the wall cavity.

Table 4. Average conditions in the indoor and outdoor rooms.

\begin{tabular}{ccccccc}
\hline $\begin{array}{c}\text { Pressure } \\
\text { Differential } \\
(\mathbf{p s f}, \mathrm{Pa})\end{array}$ & $\begin{array}{c}\text { Temperature } \\
\left({ }^{\circ} \mathrm{F}\right)\end{array}$ & $\begin{array}{c}\text { Relative } \\
\text { Humidity }(\%)\end{array}$ & $\begin{array}{c}\text { Humidity Ratio } \\
(-)\end{array}$ & $\begin{array}{c}\text { Temperature } \\
\left({ }^{\circ} \mathrm{F}\right)\end{array}$ & $\begin{array}{c}\text { Relative } \\
\text { Humidity }(\%)\end{array}$ & $\begin{array}{c}\text { Humidity Ratio } \\
(-)\end{array}$ \\
\hline $0.13(6.12)$ & 70.1 & 50.4 & 0.008 & 50.1 & 30.3 & 0.002 \\
\hline $0.23(11.0)$ & 70.1 & 50.4 & 0.008 & 50.1 & 31.2 & 0.002 \\
\hline $0.44(21.0)$ & 70.1 & 50.4 & 0.008 & 50.1 & 39.1 & 0.003 \\
\hline $0.85(40.9)$ & 70.1 & 50.4 & 0.008 & 50.1 & 48.0 & 0.004 \\
\hline $0.44(21.2)$ & 70.1 & 50.4 & 0.008 & 50.1 & 43.3 & 0.003 \\
\hline $0.24(11.3)$ & 70.1 & 50.4 & 0.008 & 50.1 & 36.7 & 0.003 \\
\hline $0.13(6.32)$ & 70.1 & 50.4 & 0.008 & 50.0 & 30.5 & 0.002 \\
\hline
\end{tabular}

Set points: $\Delta \mathrm{P}=0.1,0.21,0.42,0.84,0.42,0.21,0.1 \mathrm{psf}$; indoor room temperature $=70^{\circ} \mathrm{F}$; indoor room $\mathrm{RH}=50 \%$; outdoor room temperature $=50^{\circ} \mathrm{F}$; outdoor room $\mathrm{RH}=30 \%$. 

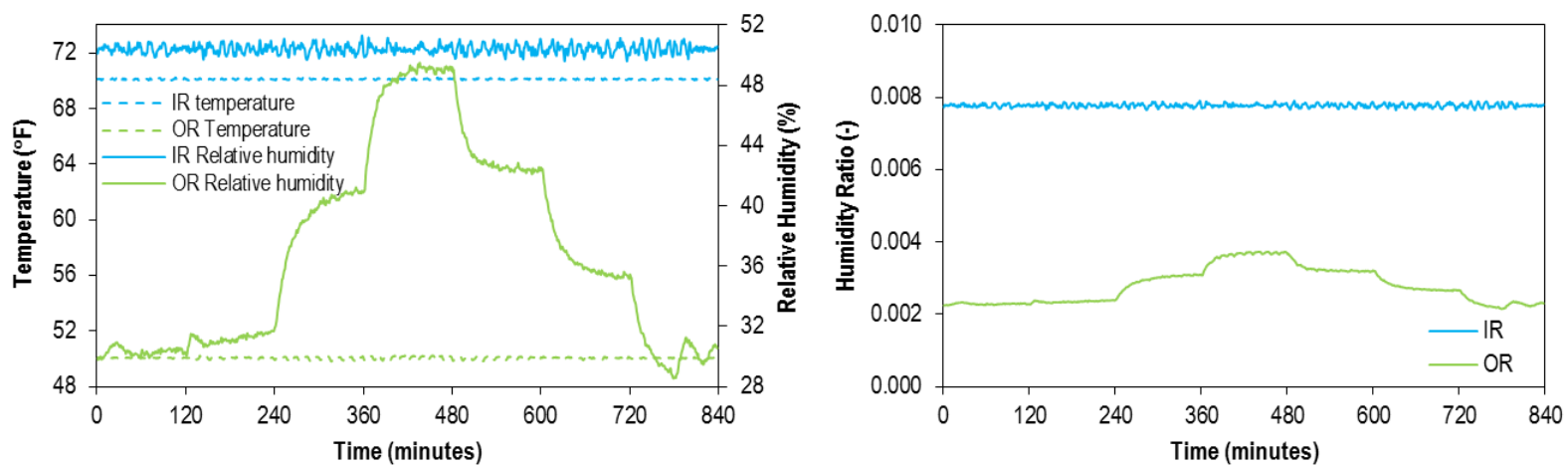

Figure 13. Conditions in the indoor room (IR) and outdoor room (OR).
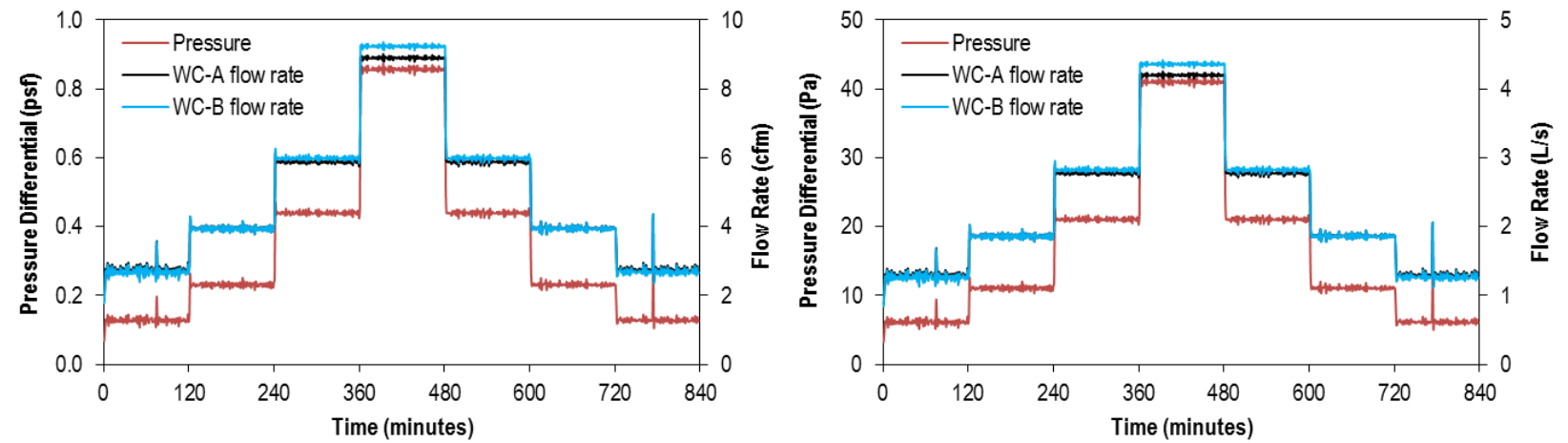

Figure 14. Pressure differentials across the test wall and flow rates through the test wall.

Figure 15 and Figure 16 illustrate the respective temperature and relative humidity data that were measured along the airflow path that laid between the fiberglass batts and the OSB, per the sensor layout in Figure 11. Temperatures within the air gap grew higher as the rate of warm air from the IR into the OR increased. These temperatures were greater at the top of the wall, where sensors were close to the air's entry point, and gradually decreased with height. Measurements typically reach steady-state in about one hour, although it took about twice as long with the highest flow rate.

In general, RH followed a trend that was opposite to what was observed with temperature: relative humidity decreased as flow rate increased and the air within the gap became warmer. This was not the case at the beginning of the test because the initial air within the gap had relatively low RH values. Moreover, this trend was less pronounced at the bottom half of the wall because the air was less effective warming up this area of the wall. Figure 16 indicates that RH was typically somewhat higher in WC-B, which is the wall cavity with the oil-based polyurethane coating on the wood members, than in WC-A. However, reaching a correlation between the coating and the RH values may not be feasible with these measurements because, as Figure 15 shows, temperatures in the air gap of WC-B also tended to be lower than in WC-A.

Measurements from other sensors in the wall are included in the Appendix A. 


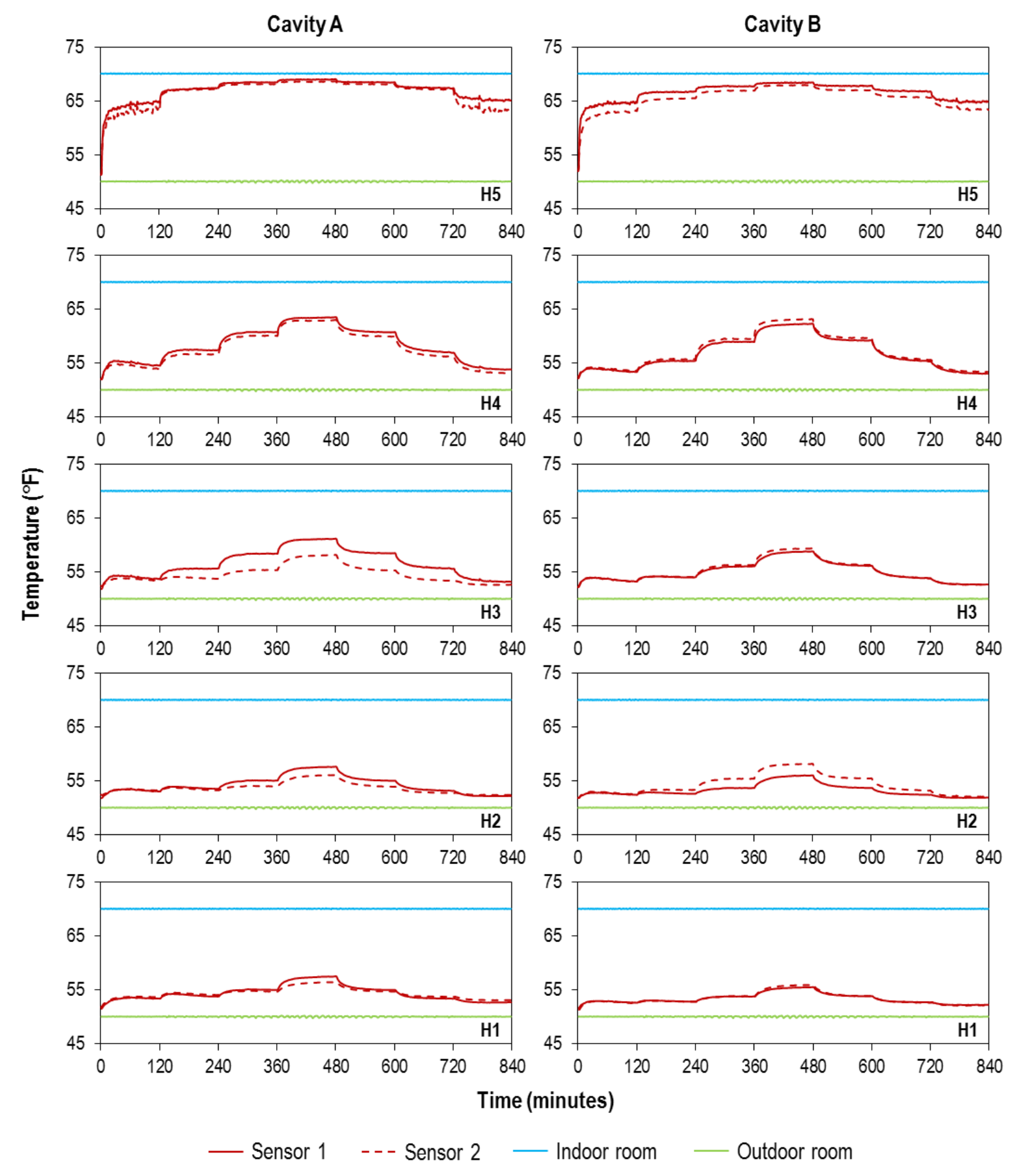

Figure 15. Temperature along the airflow path in Wall Cavities $A$ and $B$. 

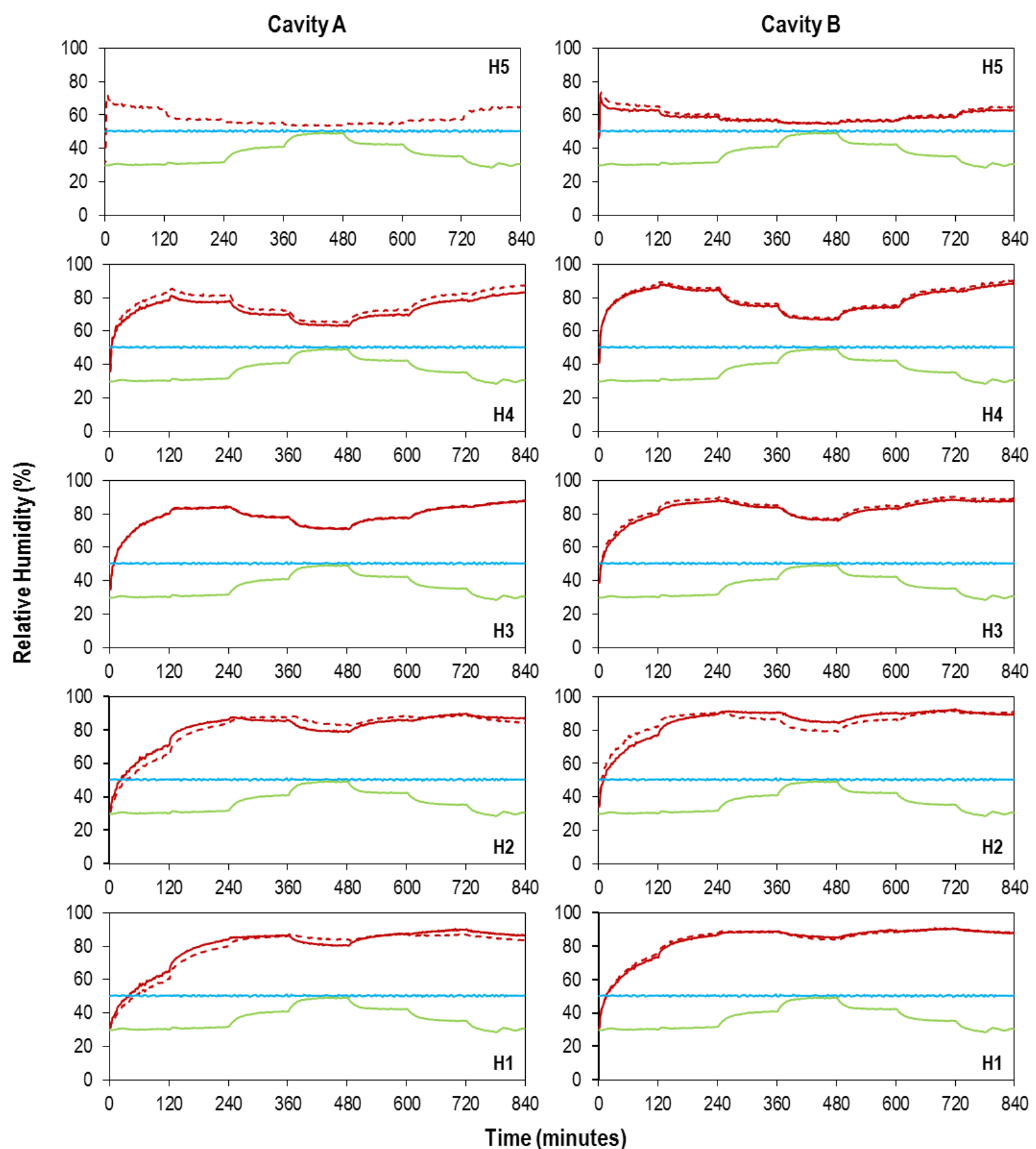

\section{Time (minutes)}

- Sensor 1 --- Sensor 2 - Indoor room - Outdoor room

Figure 16. Relative humidity along the airflow path in Wall Cavities $A$ and $B$. 

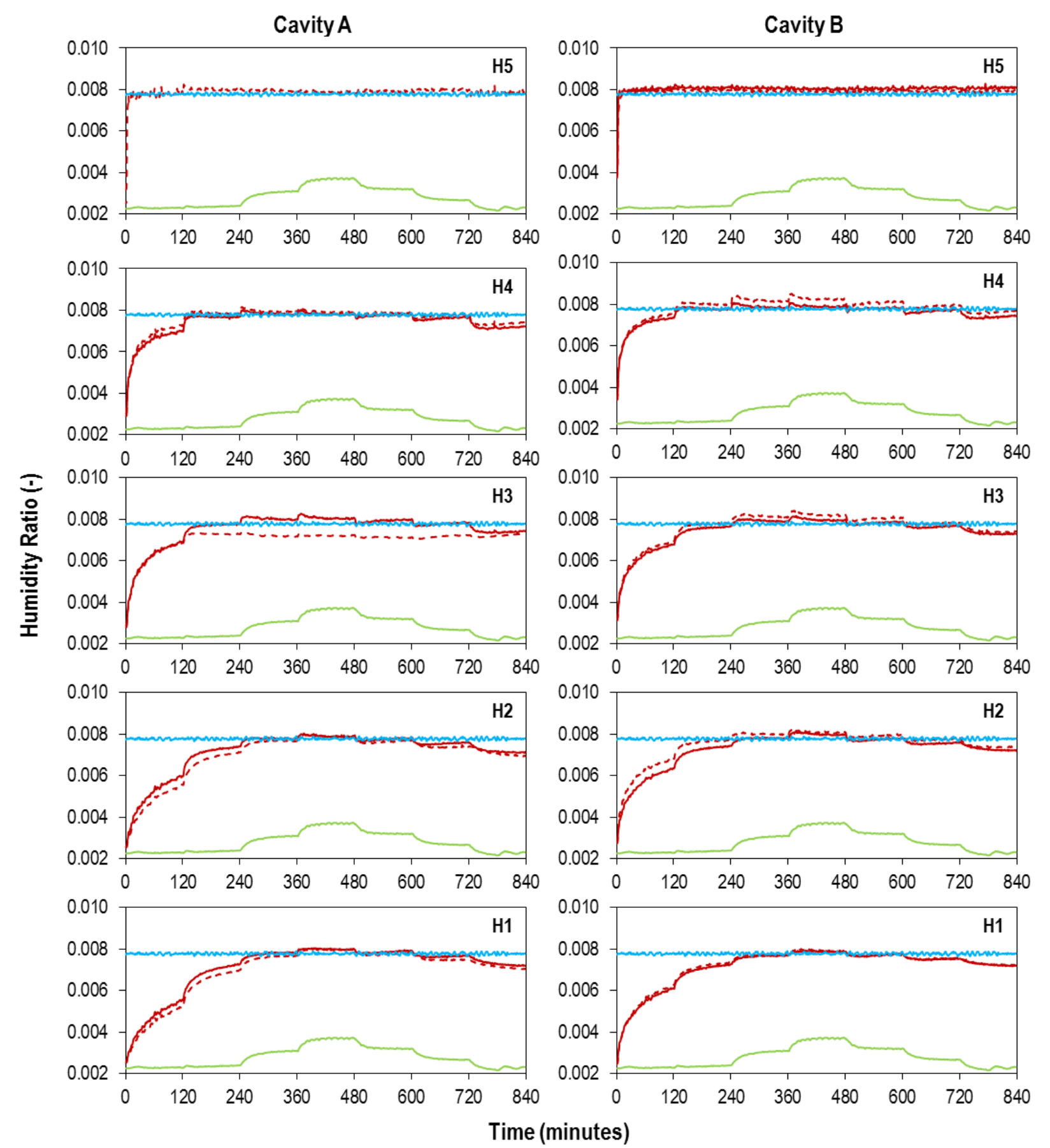

Time (minutes)

- Sensor 1 --- Sensor 2 - Indoor room - Outdoor room

Figure 17. Humidity ratio along the airflow path in Wall Cavities $A$ and $B$. 


\subsection{Initial Validation of Results}

Figure 18 shows temperatures obtained through laboratory tests and $\mathrm{WUFI}^{\circledR} 2 \mathrm{D}$ simulations, which involved the traditional approach and the proposed $\eta$-method. These temperatures were obtained at an air leakage rate of $9.1 \mathrm{cfm}(4.3 \mathrm{~L} / \mathrm{s})$, or $0.6 \mathrm{cfm} / \mathrm{ft}^{2}\left(2.9 \mathrm{~L} /\left(\mathrm{s} \cdot \mathrm{m}^{2}\right)\right)$ given that the wall cavity had was $2 \mathrm{ft}$ wide by $8 \mathrm{ft}$ tall. The four measured data points correspond to the two sensors in WC-A and WC-B. Results clearly indicate the weakness of evaluating the effects of airflow with the traditional WUFI ${ }^{\circledR} 2 \mathrm{D}$ approach: its results are on average $\pm 3.7^{\circ} \mathrm{F}$ different from the lab data. Predicting temperatures that are much lower or higher than what was observed in the lab could lead to incorrect recommendations with regard to mold growth. In contrast, results from the $\eta$-method indicated a much better agreement with the measured data, given that their difference was on average $1.3^{\circ} \mathrm{F}$.

With regard to moisture, Figure 19 suggests minimal deviations among the humidity ratio levels that were gathered through the three evaluated methods because the air's moisture content reaches an almost uniform distribution throughout the leakage path. As it was previously explained, this is because moisture exchange between the air and the materials surrounding the leakage path is a very slow process. Consequently, results indicate that the $\eta$-method has higher importance when estimating temperature than moisture levels.

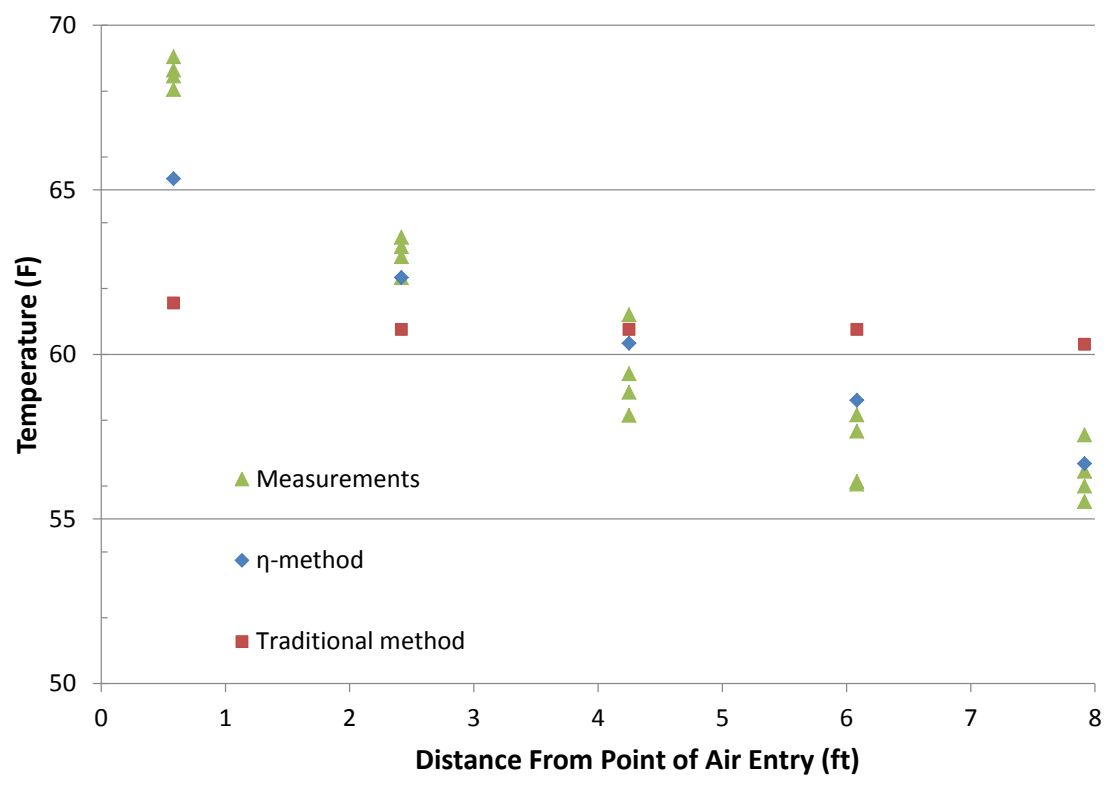

Figure 18. Simulated vs. measured steady-state temperatures at a leakage rate of $9.1 \mathrm{cfm}(4.3 \mathrm{~L} / \mathrm{s})$. 


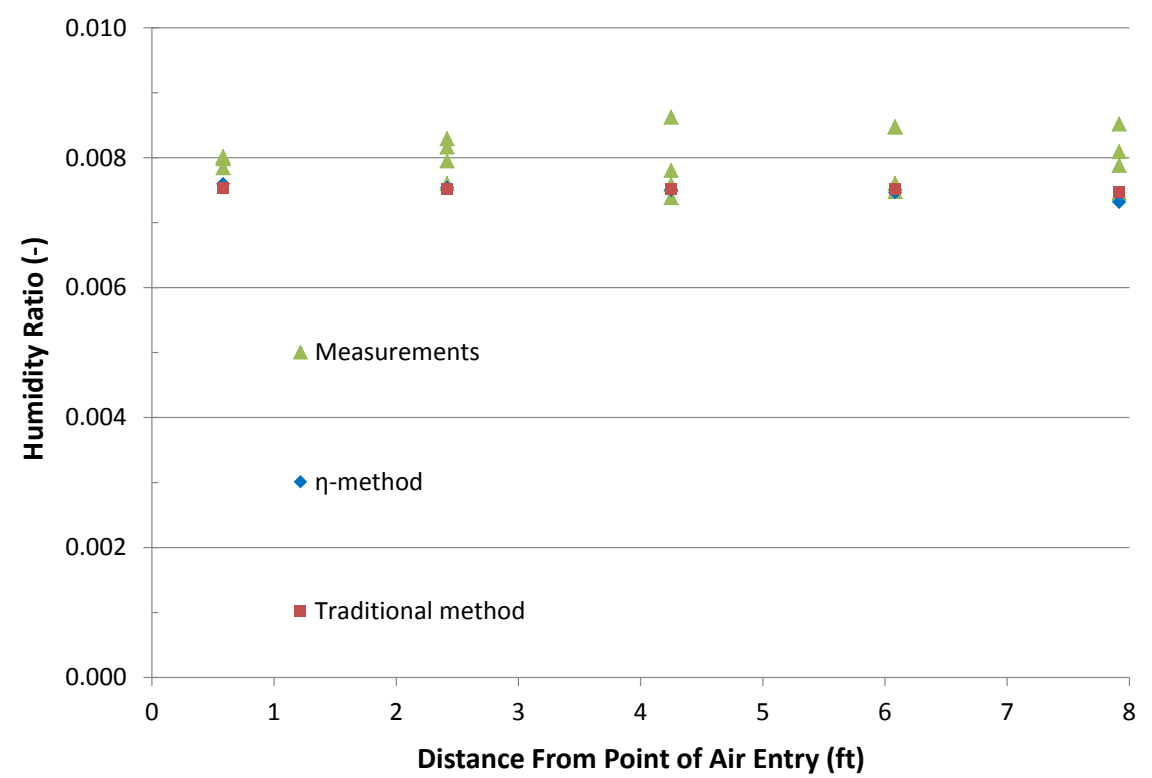

Figure 19. Simulated vs. measured stead-state humidity ratio levels at a leakage rate of $9.1 \mathrm{cfm}(4.3 \mathrm{~L} / \mathrm{s})$.

Figure 20 shows how the mathematical solution behind the $\eta$-method matches the temperatures obtained through laboratory tests at a leakage rate of $5.8 \mathrm{cfm}(2.7 \mathrm{~L} / \mathrm{s})$ or $0.4 \mathrm{cfm} / \mathrm{ft}^{2}\left(1.8 \mathrm{~L} /\left(\mathrm{s} \cdot \mathrm{m}^{2}\right)\right)$.

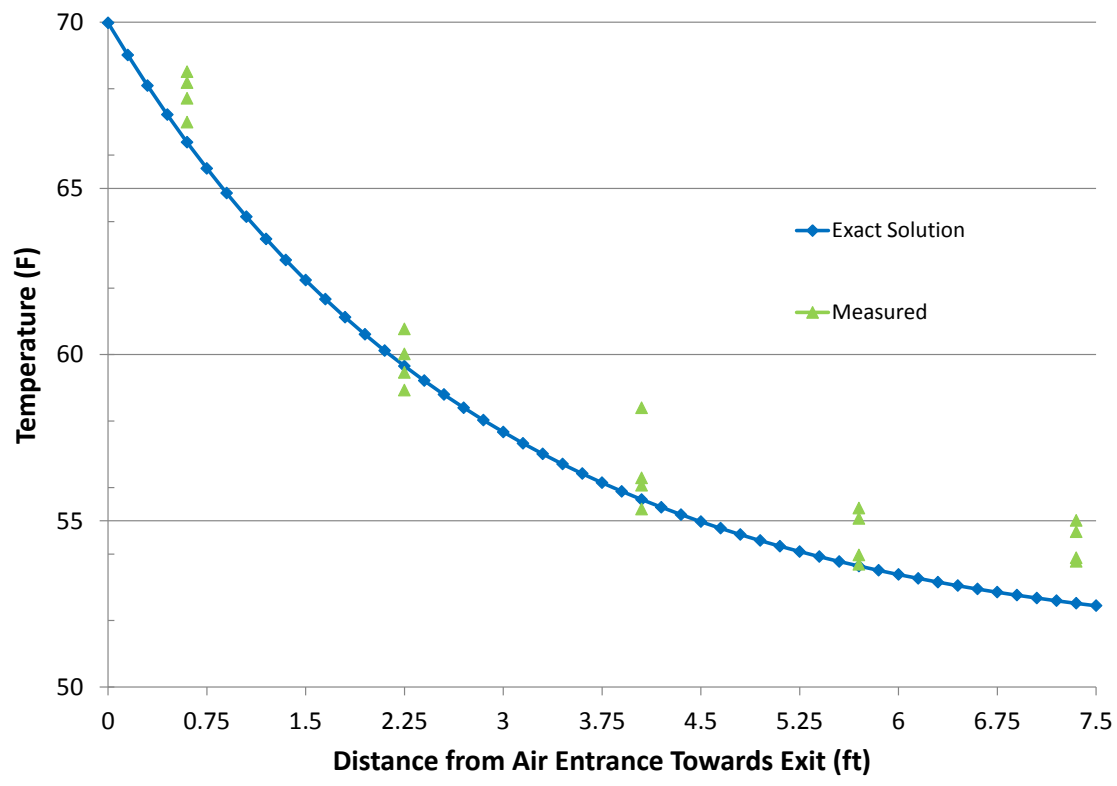

Figure 20. Calculated vs. measured steady-state temperatures at a leakage rate of $5.8 \mathrm{cfm}(2.7 \mathrm{~L} / \mathrm{s})$.

\subsubsection{Implementation of Calibrated Simulation Tool}

Two simulation models were created, one for Climate Zone 2 (CZ2) and one for Climate Zone 5 (CZ5). The simulation model for CZ5 utilized the wall assembly that was used to calibrate WUFI ${ }^{\circledR}$ : vinyl siding, mechanically-fastened water-resistive barrier (58 perms), 7/16" oriented strand board (OSB), $2 \times 6$ studs at 24" on center, R19 kraft-faced fiberglass insulation, and drywall. Additionally, it included an air leakage 
path between the OSB and fiberglass batts through which air leakage occurred. The simulation model for CZ2 differed slightly because kraft paper was not applied on the inside of the insulation given that it is not required by the 2012 IRC in this climate. Also, air leakage occurred through an air leakage path that was located between the drywall and the insulation; these conditions are more prone to moisture problems in the hot-humid climates.

The simulations for CZ2 and CZ5 were conducted with probabilistically varying airtightness levels; this type of statistical information is needed in order to be able to perform a probabilistic assessment. ACH50 values were obtained from the Residential Diagnostics Database (LBL 2014), which represent the air changes per hour or airtightness of a whole building enclosure at a pressure differential of $50 \mathrm{~Pa}$. Assumptions that were made to estimate these data were: $2400 \mathrm{ft}^{2}$ floor area, $8 \mathrm{ft}$ ceiling height, built after 2000, Energy Star certified, unconditioned basement or vented crawlspace, ducts in an unconditioned attic or basement, and house located in a humid region. The data in Figure 21 are presented as a cumulative distribution function, and the listed values are the ACH50 data at a specific percentile, $p$, in Climate Zones 2 and 5.

In order to make the statistical data applicable to the simulation model, an airtightness specific air leakage coefficient, $C_{p}$, was expressed for each value of ACH50 presented in Figure 21. This was made possible through the following relationships:

$$
\begin{aligned}
& Q=C \cdot \Delta \mathrm{P}^{n} \\
& Q 50_{p}=A C H 50_{p}=C_{p} \cdot \Delta \mathrm{P}^{n}
\end{aligned}
$$

where $C_{p}$ was expressed with the assumption of an air pressure exponent, $n$, of 0.65 .

$$
C_{p}=\frac{Q_{p}(\Delta P)}{\Delta \mathrm{P}^{n}}=\frac{A C H 50_{p}}{50^{0.65}}
$$

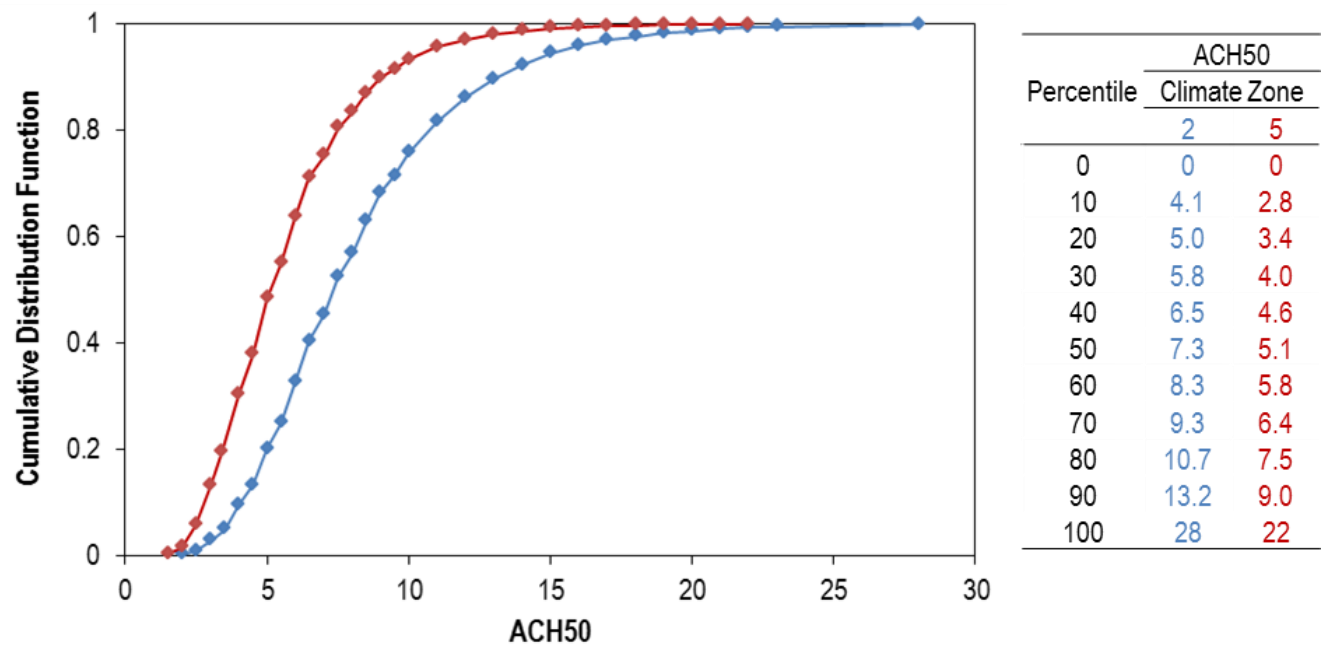

Figure 21. Air changes per hour at a pressure differential of 50 Pa (ACH50) in Climate Zones 2 and 5 (LBL 2014). Specific levels of ACH50 are given for every tenth percentile.

Consequently, the calculated air leakage coefficient, $C_{p}$, corresponds to a specific percentile and remains constant throughout the simulation for that percentile. In order to estimate hourly variations of air leakage rate, and to be able to use Eq. (18b) for arbitrary cases, a pressure difference, $\Delta P$, over the simulated wall was needed. Hourly-varying wind-induced pressure differences were defined for Chicago (Climate 
Zone 5) and Houston (Climate Zone 2) based on the climate files from WUFI ${ }^{\circledR} 2 \mathrm{D}$. The relationship between wind speed and the induced pressure on a wall surface was estimated using the ASCE/SEI 7-10 Standard (2010) in which the air pressure, $P_{\text {wind }}\left(\mathrm{lb} / \mathrm{ft}^{2}\right)$, acting on the wall surface is expressed as

$$
P_{\text {wind }}=0.00256 \cdot \dot{V}^{2}=\Delta P
$$

where $\dot{V}$ is the wind speed (mph). Naturally, the direction of $\dot{V}$ will influence $P_{\text {wind }}$. However, directional adjustments for the wind speed were not done in this study; therefore, the results that are presented in this report should be considered as a worst-case scenario.

Hourly values for $Q_{p}$ were then calculated using the wind pressures obtained from Eq. (20) and air leakage rates were calculated using Eq. (18a). However, one more step was needed to create the inputs for WUFI $^{\circledR} 2 \mathrm{D}$ that represent the air leakage. $Q_{p}$ was then expressed in $\mathrm{ACH}$, as seen in the details of Eq. (11) in Section 4.3.1.2.

$$
A C H_{p}=Q_{p} \cdot \frac{V}{t}
$$

where $t$ (s) equals 60 if $Q_{p}$ is expressed in cfm, or 3600 if $Q_{p}$ is expressed in $\mathrm{ft}^{3} / \mathrm{s}$.

Finally the prosed $\eta$-method is adopted by correcting the $\mathrm{ACH}$ for each hour to take into account the geometry of the air leakage path. Consequently, $\eta$ value from Eq. (10) calibrates the ACH through a calibrated air change rate, $\mathrm{ACH}_{n}$ :

$$
A C H_{\eta, p}=\eta(x, Q) \cdot A C H_{p}
$$

In addition, two levels of indoor moisture supply were implemented into the simulation model from ASHRAE 160 (ASHRAE 2011); one for 2 bedrooms and an ACH of 0.6 and for 6 bedrooms with an ACH of 0.2 .

\subsubsection{Analysis of Results}

Temperature, relative humidity, humidity ratio, and mold growth were evaluated along the air leakage path of the two simulation models. Results covered in this report focus on RH and mold growth at two locations along the flow path: near the entrance and near the exit of the inflowing air as indicated in Figure 22. Also, only results from simulations with high indoor moisture sources are presented because the low sources did not display moisture risks.

Note that the following simulation results should be interpreted with caution because of the following assumptions:

- Airtightness was the only factor that was varied in a probabilistic manner.

- Wind pressures that were derived from the weather files were applied as a unidirectional and normal force on the wall. Therefore, airflows did not account for changes in wind direction and were simulated as either infiltration or exfiltration.

- Air leakage was uniformly distributed over the entire surface area of exterior walls; while in reality, areas without penetrations will likely experience lower flow rates. 
Because the last two items lead to very conservative results, the simulation results that follow should only be used as an example of the implementation of the durability protocol and not as precise findings. Both these items will be resolved in future analyses, which will provide more realistic results.

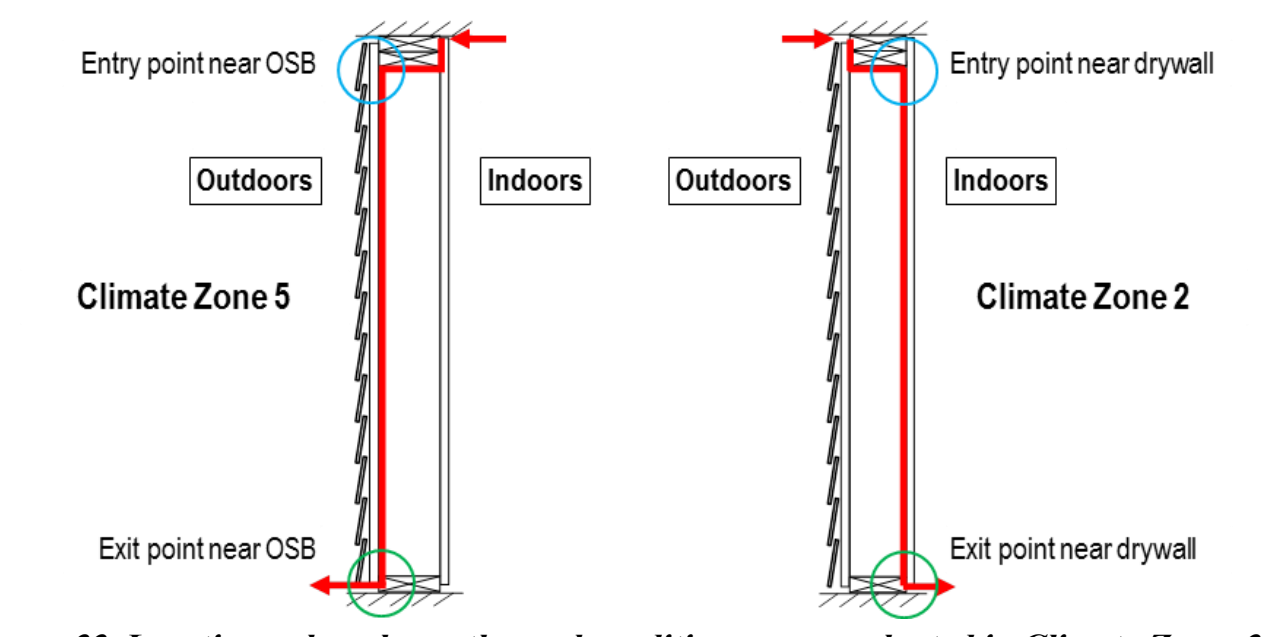

Figure 22. Locations where hygrothermal conditions were evaluated in Climate Zones 2 and 5.

Figure 23 and Figure 24 correspond to the 2-year relative humidity analyses in CZ5. Figure 23 shows modeled RH at the OSB near the entrance of the leakage path for four scenarios with different airtightness or ACH50 levels. The green curve illustrates the results from a simulated wall with an airtightness of 2.8 ACH50, which is specific to the $10^{\text {th }}$ percentile of the homes that were built after 2000 in CZ5. The blue, yellow and red curves represent the ACH50 values given by the $40^{\text {th }}, 70^{\text {th }}$ and the $100^{\text {th }}$, respectively. Results indicate that all of these flow rates generated $\mathrm{RH}$ values that were higher than the recommended maximum of $80 \%$ for about half of the year. Moreover, only the highest flow rate did not lead to condensation because it was able to maintain the surface of the OSB at a temperature higher than its dew point. Modeled RH values were similarly high at the exit point of the leakage path. However, the higher flow rates were more conducive to condensation and elevated $\mathrm{RH}$ because increases in flow carry more moisture and because temperatures near the exit are lower. 


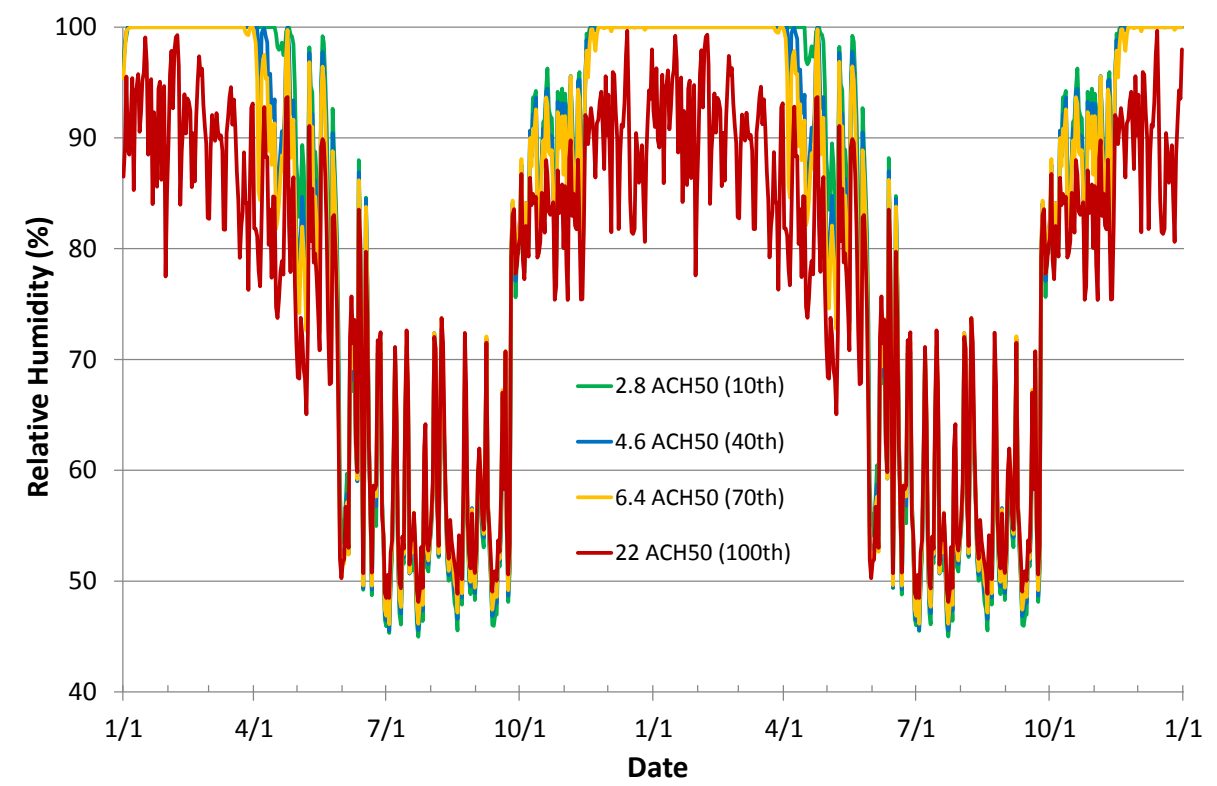

Figure 23. Relative humidity at OSB near the air's entry point in a wall in Climate Zone 5 and with high indoor moisture sources. The curves represent the results from a simulated wall with four different airtightness values (ACH50).

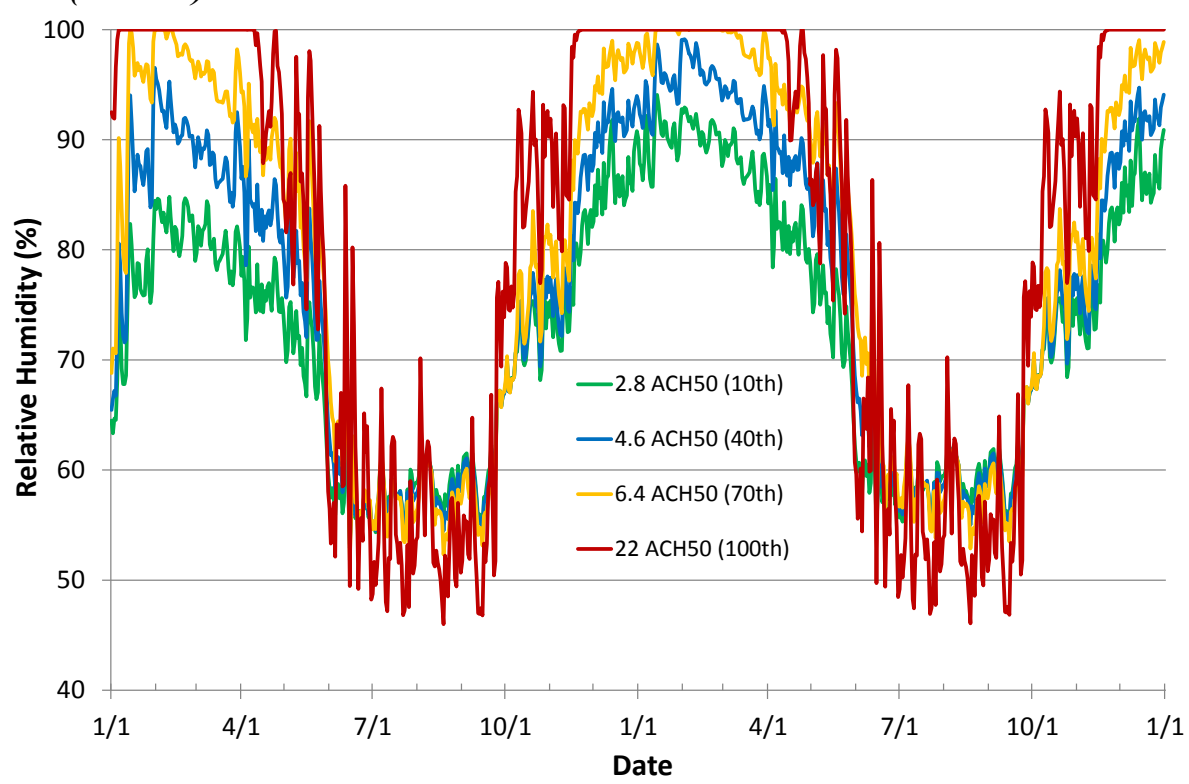

Figure 24. Relative humidity at OSB near the air's exit point in a wall in Climate Zone 5 and with high indoor moisture sources. The curves represent the results from a simulated wall with four different airtightness values (ACH50).

Figure 25 and Figure 26 illustrate the results for CZ2. Here, the data were gathered at the drywall surfaces that were near the air's entry and exit points, respectively. As with the data from CZ5, relative humidity levels were the highest near the air leakage entrance. However, not much of a difference was noted at this location due to flow rate, and RH was greater than $80 \%$ for short periods of time. Variations in relative humidity were more significant near the exit of the air leakage path. According to Figure 26, higher RH was encountered with increases in air leakage. 


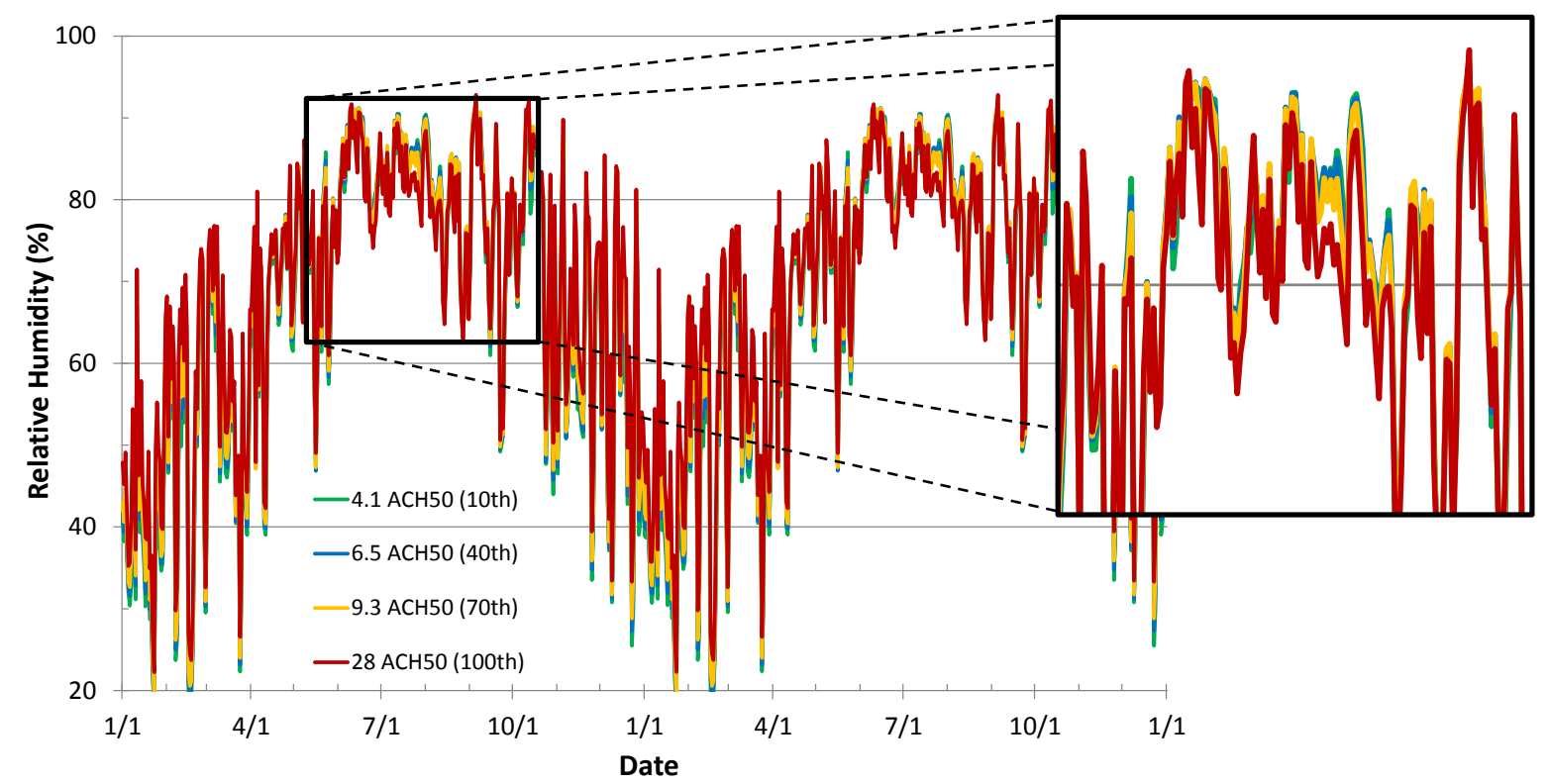

Figure 25. Relative humidity at drywall near the air's entry point in a wall in Climate Zone 2 and with high indoor moisture sources. The curves represent the results from a simulated wall with four different airtightness values (ACH50).

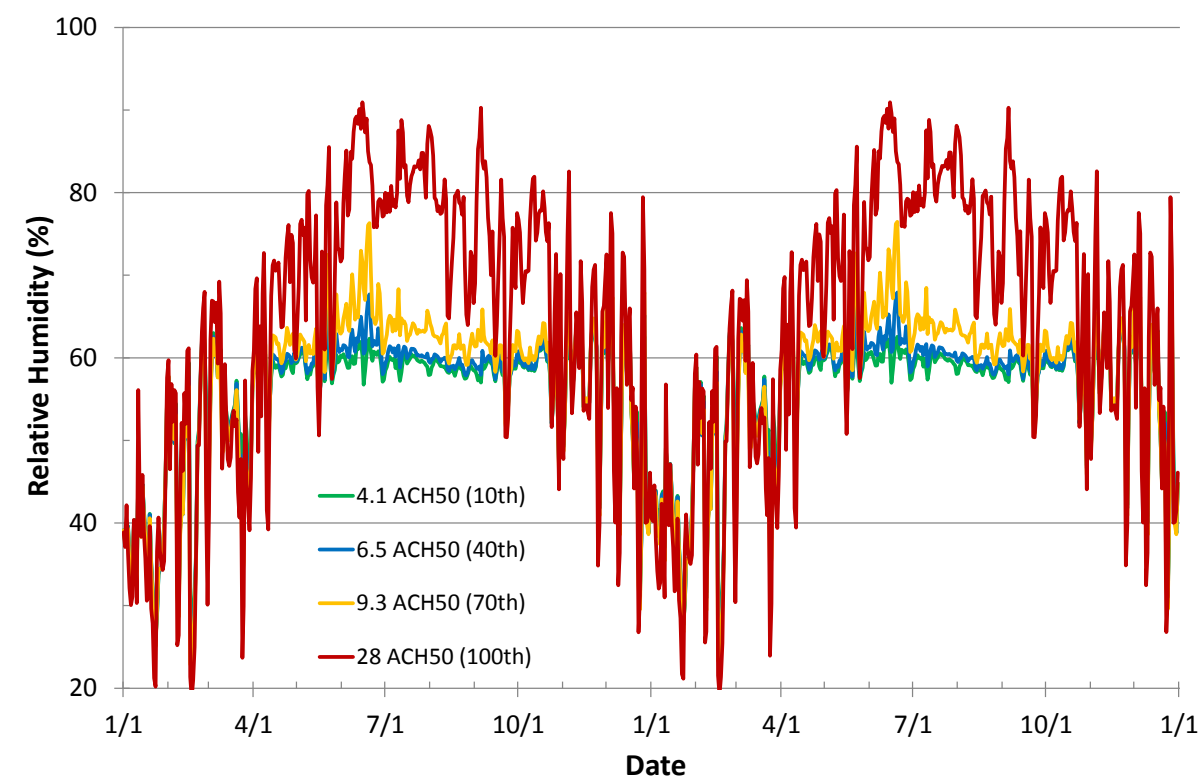

Figure 26. Relative humidity at drywall near the air's exit point in a wall in Climate Zone 2 and with high indoor moisture sources. The curves represent the results from a simulated wall with four different airtightness values (ACH50).

More results from the 2-year simulations are presented in Appendix B.

Figure 27 and Figure 28 depict how the mold growth index level changed over a period of two years in the wall assembly in CZ5. Apparently, the highest risk occurs in locations close to where the air enters the air leakage path with most of the ACH50 values. For locations farther away from the entrance, the risk seems to increase with higher flow rates. Since the risk of mold is high even at low air flow rates, as seen in Figure 27, it appears that the critical air flow rate should be based on results that were obtained 
near the entrance of the flow path. This conclusion is in accordance with what was observed with the relative humidity levels. Note that in both Figure 27 and Figure 28, the mold growth index levels seem as if they will continue to increase with time, which indicates that the highest risk of mold is yet to be seen.

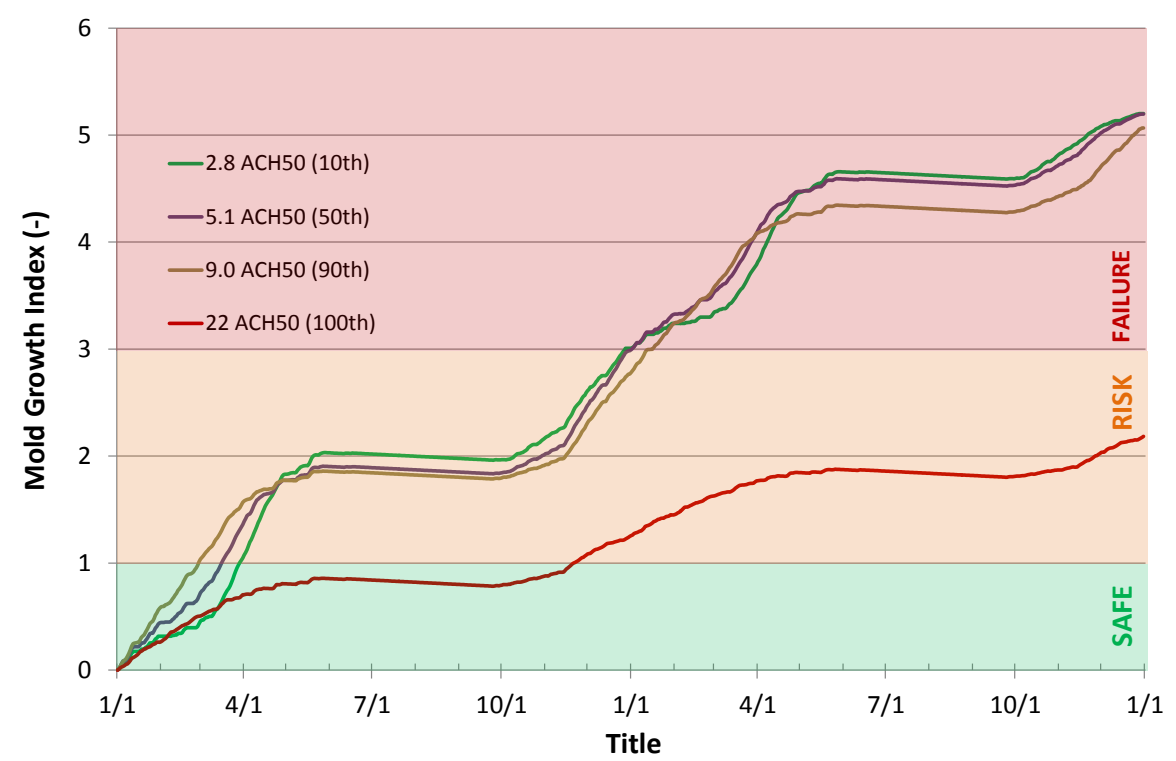

Figure 27. Mold growth index at OSB near the air's entry point in a wall in Climate Zone 5 and with a high indoor moisture supply.

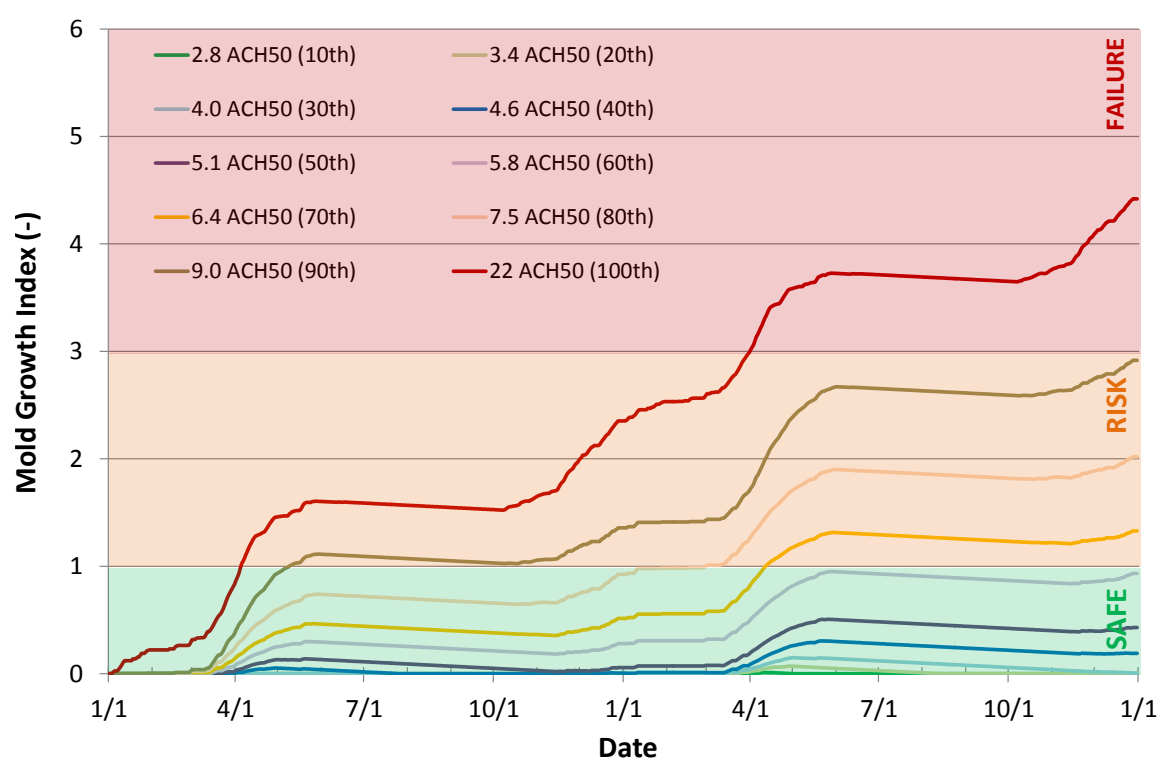

Figure 28. Mold growth index at OSB near the air's exit point in a wall in Climate Zone 5 and with a high indoor moisture supply.

In CZ 2, the risk of mold growth is much lower than for the simulated scenarios in CZ 5. Figure 29 and Figure 30 show the risk of mold growth on the exterior side of the drywall to be low. However, there seems to be a slight increase in the mold growth index near the air's exit point, which indicates that simulations beyond two years should be conducted. 
Figure 31 provides an example of how the proposed protocol will generate results to identify wall assemblies that are moisture durable and energy efficient. This figure summarizes a small fraction of the simulation results, which suggests that the likelihood that the scenario that was evaluated in CZ2 will have mold growth problems is very low. However, a critical airflow rate was observed in the scenario that was studied in CZ5, where walls with leakage rates that are lower than $Q_{c r}$ will be less likely to encounter moisture durability problems.

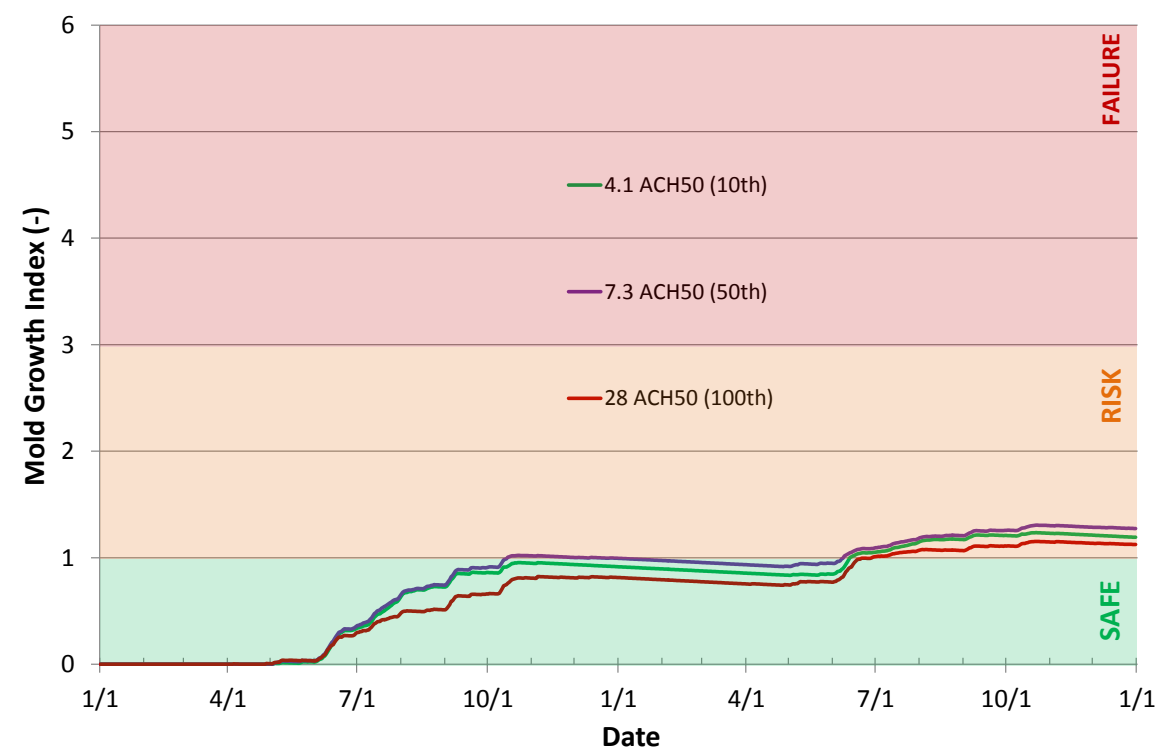

Figure 29. Mold growth index at drywall near the air's entry point in a wall in Climate Zone 2 and with a high indoor moisture supply.

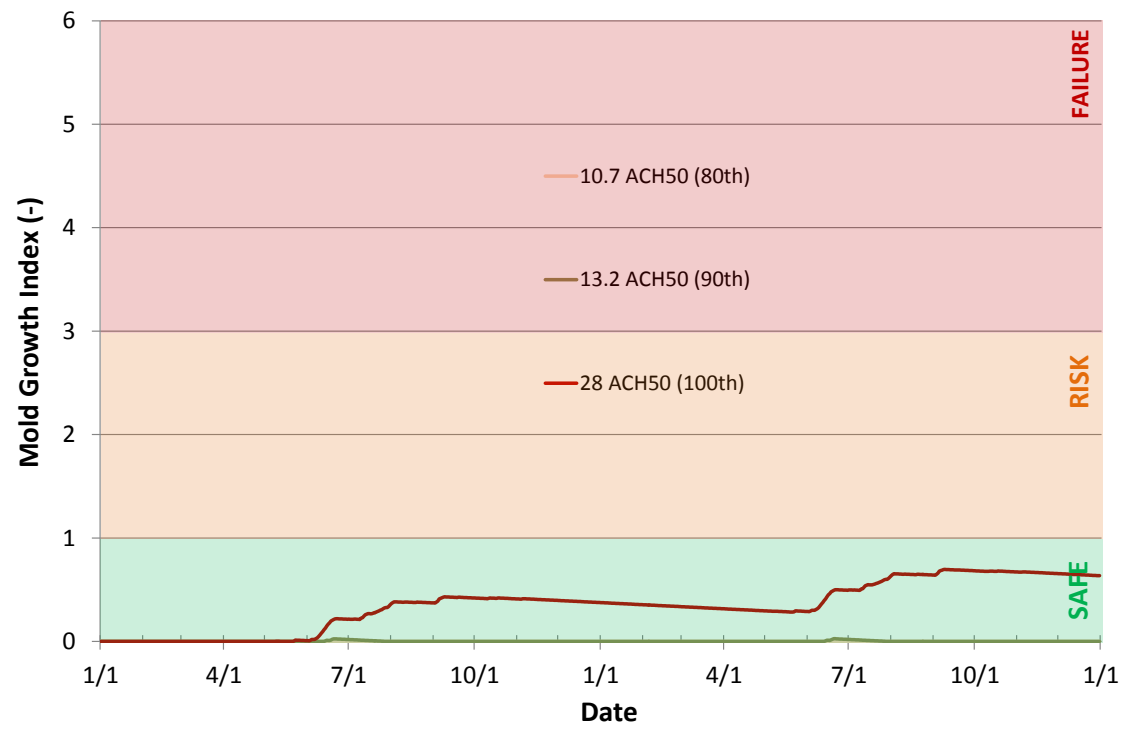

Figure 30. Mold growth index at drywall near the air's exit point in a wall in Climate Zone 2 and with high a indoor moisture supply. 


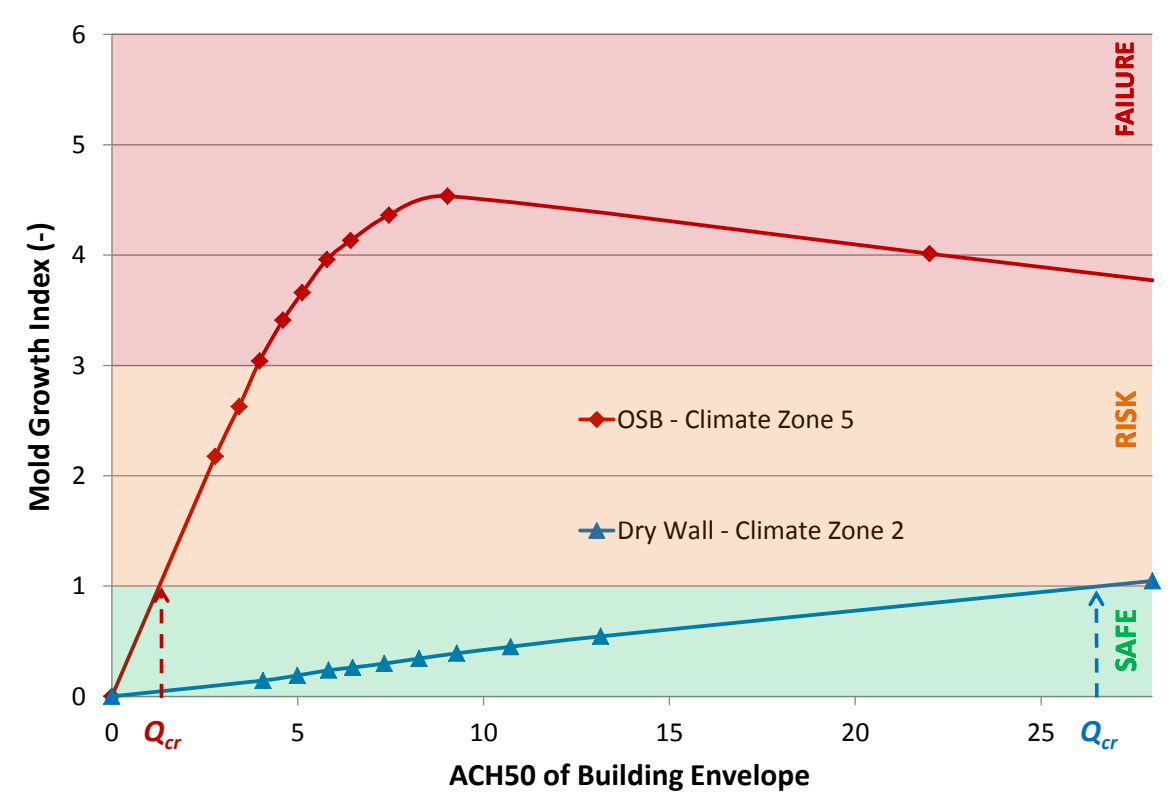

Figure 31. Mold growth index as a function of $\mathrm{ACH} 50$.

\subsubsection{Validation of Simulations through Laboratory and Field Tests}

As indicated in Section 3.3.1.3, the initial validation of the $\eta$-method appears to suggest promising results. Further laboratory experiments with the current test wall include evaluating lower flow rates, different indoor and outdoor environmental conditions, infiltration, and exterior continuous insulation. Thereafter, experiments will be repeated with a different wall assembly. Findings will be used to further validate and calibrate the simulation tool. Subsequently, the improved software will be utilized to conduct stochastic analyses that will identify various energy-efficient wall assemblies that have a low probability of encountering moisture durability problems. Based on this information, designs will be selected to be assessed through field tests, which will provide additional data that will strengthen the simulation and laboratory results.

\subsection{Recommendations Based on Expected Hygrothermal Performances}

This section serves to evaluate the results from simulations and measurements that were conducted. Since this study was intended to be an example of the proposed moisture durability protocol, the outcome is not as relevant as the described procedure. Therefore, recommendations are not provided at this time. 


\section{CONCLUSION}

The work described in this report was the initial step in a multi-year project that will provide energyefficient and moisture-durable wall designs to the America residential building community. To this end, a probabilistic approach was targeted in order to take into account numerous variables, and therefore, expand the credibility and applicability of the results. The main initial task was to propose and verify the feasibility of a moisture durability protocol that would enable the hygrothermal risk assessment of wall assemblies. This report covers an example of the implementation of the proposed protocol with a 2012 IECC compliant wall assembly. Significant accomplishments include:

- Successful implementation of the proposed protocol through a case study;

- Development of the $\eta$-method that improves $\mathrm{WUFI}^{\circledR} 2 \mathrm{D}$ since this simulation tool has limited capabilities to address airflow, although air leakage is one of the most significant causes of moisture problems in building components;

- Initial validation of the $\eta$-method where preliminary results show significant improvements over the traditional way in which $\mathrm{WUFI}^{\circledR} 2 \mathrm{D}$ calculates the temperature distribution in an air leakage path; and

- Simulation results appear to indicate that the improved WUFI ${ }^{\circledR} 2 \mathrm{D}$ can be used to estimate the critical air leakage rate above which the probability to encounter moisture durability problems in a specific wall assembly increases. This information will be valuable when trying to determine which wall assemblies exhibit higher tolerance toward airflow. Furthermore, this knowledge will be welcomed by building codes as they set the newly required air leakage rates.

Upcoming tasks include continuing further validation and calibration of the $\eta$-method with laboratory tests that incorporate more environmental conditions and wall assemblies. These will be followed by stochastic analyses that will identify energy-efficient wall assemblies with a low probability of encountering moisture problems, and by field tests that will strengthen the simulation and laboratory results. This robust evaluation process will facilitate the adoption rate of energy-efficient wall assemblies by providing conclusive data on moisture durable designs.

\section{ACKNOWLEDMENTS}

This work was supported by the Assistant Secretary for Energy Efficiency and Renewable Energy, Office of the Building Technology Program, U.S. Department of Energy, under Contract No. DE- AC0500OR22725. The authors are especially grateful to Manfred Kehrer for enabling WUFI ${ }^{\circledR} 2 \mathrm{D}$ to conduct multiple simulation runs, and thankful to André Desjarlais and Philip Boudreaux for reviewing this report. 


\section{REFERENCES}

ASCE/SEI 7-10. 2010. Minimum design loads for buildings and other structures. ASCE/SEI 7-10: American Society of Civil Engineers.

ASHRAE. 2009. Criteria for moisture-control design analysis in buildings. ANSI/ASHRAE Standard 160-2009. Atlanta, GA: American Society of Heating, Refrigerating and Air-Conditioning Engineers, Inc.

ASHRAE. 2013a. Energy standard for buildings except low-rise residential buildings. ANSI/ASHRAE Standard 90.1-2013. Atlanta, GA: American Society of Heating, Refrigerating and Air-Conditioning Engineers, Inc.

ASHRAE. 2013b. Handbook - Fundamentals. Atlanta, GA: American Society of Heating, Refrigerating and Air-Conditioning Engineers, Inc.

ASTM International. 2004. ASTM E283. Standard test method for rate of air leakage through exterior windows, curtain walls, and doors under specific pressure differentials across the specimen. Pennsylvania: American Society for Testing and Materials International.

Cornick SM. 2008. Task 5: Proposed test protocol for walls of houses in extreme cold regions. Part 1: Defining exterior conditions. National Research Council Canada.

Desmarais G, Derome D, Fazio P. 2000. Mapping of air leakage in exterior wall assemblies. J Bldg Physics 24:132-154.

Fisk WJ, Lei-Gomez Q, Mendell MJ. 2007. Meta-analyses of the associations of respiratory health effects with dampness and mold in homes. Indoor Air 17(4):284-296.

Fox MJ. 2014. Hygrothermal performance of highly insulated wood frame walls with air leakage: field measurements and simulations. M.A.Sc. Thesis. Ryerson University, Toronto, Canada. Report B-1239.5.

Glass SV. 2013. Hygrothermal analysis of wood-framed wall assemblies in a mixed-humid climate. Madison, WI: US Department of Agriculture, Forest Service, Forest Products Laboratory. Research paper FPL-RP-675.

Hagentoft C. 2001. Introduction to building physics. Studentlitteratur, Sweden.

Holm A. 2001. Ermittlung der Genauigkeit von instationären hygrothermischen Bauteilberechnungen mittels eines stochastischen Konzeptes. (Doctoral), Univ. Stuttgart.

Home Innovation Research Labs. 2014. Moisture performance of walls in energy efficient homes. Home Innovation Research Labs. Report 5932.001_08142014.

Hukka A, Viitanen H. 1999. A mathematical model of mould growth on wooden materials. Wood Science and Technology 33(6):475-85.

IECC. 2012. 2012 International Energy Conservation Code. Country Club Hill, IL: International Code Council.

IRC. 2012. 2012 International Residential Code. Country Club Hill, IL: International Code Council.

Kehrer M, Schmidt T. 2008. Radiation effects on exterior surfaces. In: Proceedings from the Nordic Symposium on Building Physics. Copenhagen, 2008.

Künzel HM. 1995. Simultaneous heat and moisture transport in building components: one- and twodimensional calculation using simple parameters. Stuttgart, Germany: Fraunhofer IRB Verlag. 
Künzel HM, Zirkelbach D, Schafaczek B. 2011. Vapour control design of wooden structure including moisture sources due to air exfiltration. In: Proceedings from the $9^{\text {th }}$ Nordic Symposium on Building Physics, NSB 2011. Tampere, Finland, May 29 - June 2, 2011.

Langmans J, Klein R, Roels S. 2012. Hygrothermal risks of using exterior air barrier systems for highly insulated light weight walls: a laboratory investigation. Buildings and Environment 56:192-202.

LBL (Lawrence Berkeley Laboratory). 2014. Residential Diagnostics Database. Available: http://resdb.lbl.gov/main.php?step=2\&sub=1\&run_env_model=\&dtype1=\&dtype2=\&is_ca=\&calc_id=2 $\&$ floor_area $=1500 \&$ house_height $=8 \&$ year_built $=1 \&$ wap $=0 \&$ ee_home $=0 \&$ region $=1 \&$ zone $=1 \&$ foundatio $\mathrm{n}=1 \&$ duct $=1 \#$ [accessed Nov 2014].

Lepage R, Schumacher C, Lukachko A. 2013. Moisture management for high r-value walls. Building Science Corporation, Report 1319, prepared for the National Renewable Energy Laboratory.

Mendell MJ, Mirer AG, Cheung K, Tong M, Douwes J. 2011. Respiratory and allergic health effects of dampness, mold, and dampness-related agents: A review of the epidemiologic evidence. Environmental Health Perspectives 119(6):748-756.

Merill JL, TenWolde A. 1989. Overview of moisture-related damage in one group of Wisconsin manufactured homes. ASHRAE Transactions 95(1):405-414.

Ojanen T, Kohonen R, Kumaran M. 1994. Modeling heat, air and moisture transport through building materials and components. In: Trechsel, HR ed., Moisture control in buildings, $1^{\text {st }}$ ed. West Conshohocken, PA: ASTM International: 18-34. Chapter 2.

Ojanen T, Viitanen H, Peuhkuri R, Lahdesmaki K, Vinha J, Salminen K. 2010. Mold growth modeling of building structures using sensitivity classes of materials. In: Proceedings from the Buildings XI Conference. Clearwater, FL, December 2010.

Pallin SB, Kehrer M. 2013. Condensation Risk of Mechanically Attached Roof Systems in Cold Climate Zones. In: Proceedings from the RCI 28th International Convention \& Trade Show. Orlando, FL, March 14-19, 2013.

Straube J, Smegal J. 2012 revision. Building America special research project: High-r walls case study analysis. Building Science Corporation, Report 0903, prepared for the National Renewable Energy Laboratory. 


\section{APPENDIX A: LABORATORY DATA}

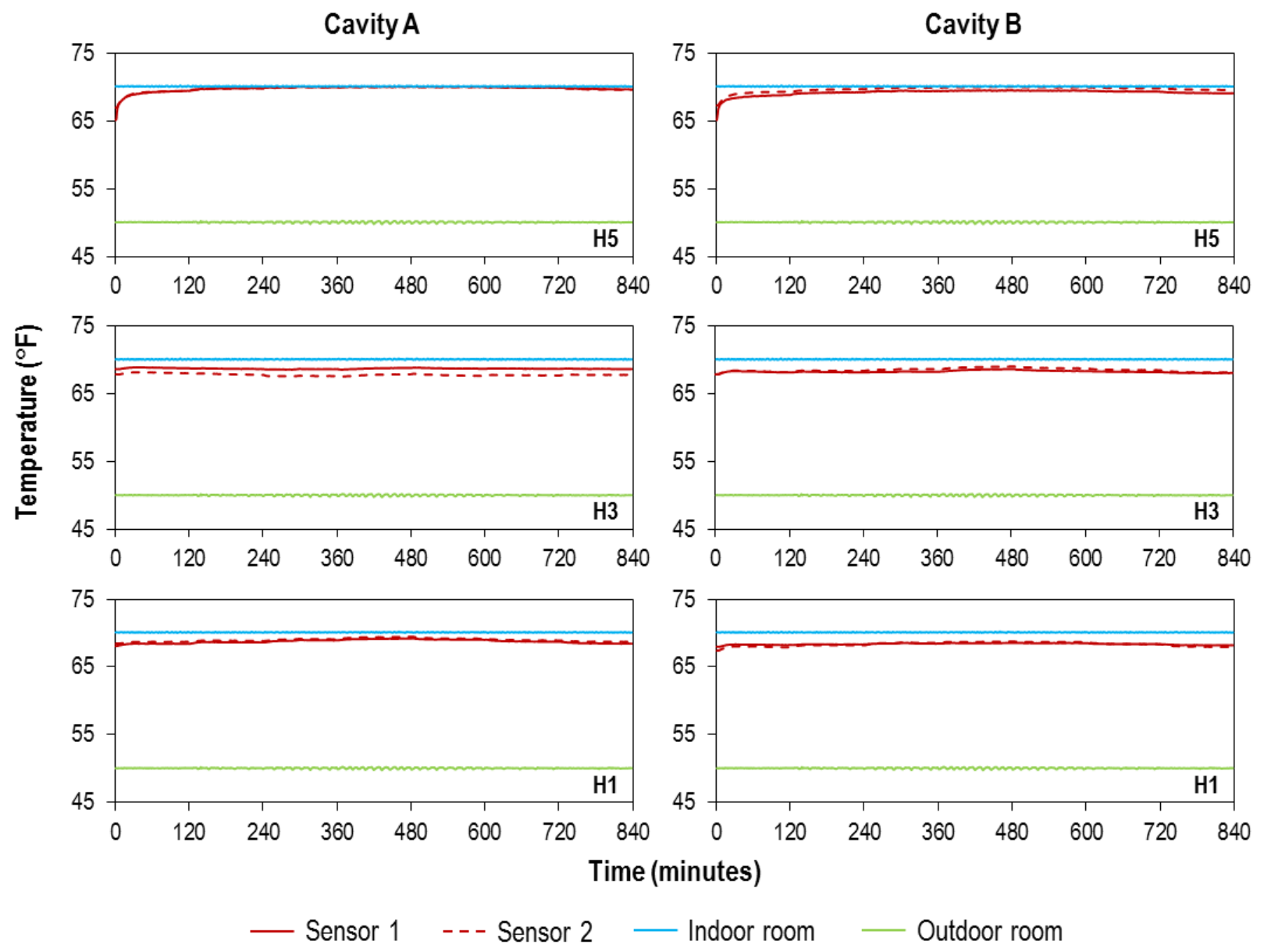

Figure A 1. Temperature at exterior side of the drywall in Wall Cavities $A$ and $B$. 

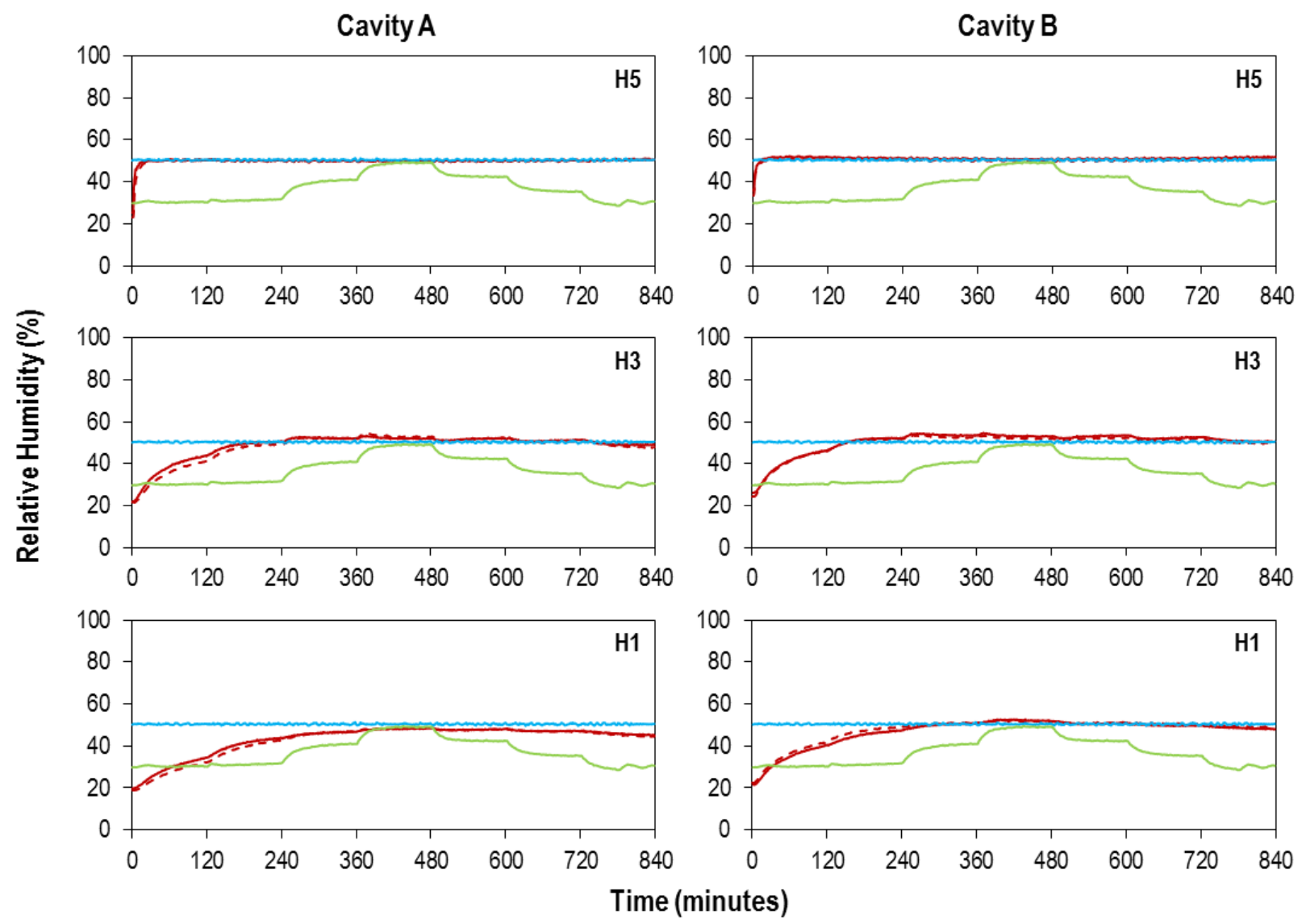

Sensor 1 --- Sensor 2 - Indoor room - Outdoor room

Figure A 2. Relative humidity at exterior side of the drywall in Wall Cavities $A$ and $B$. 


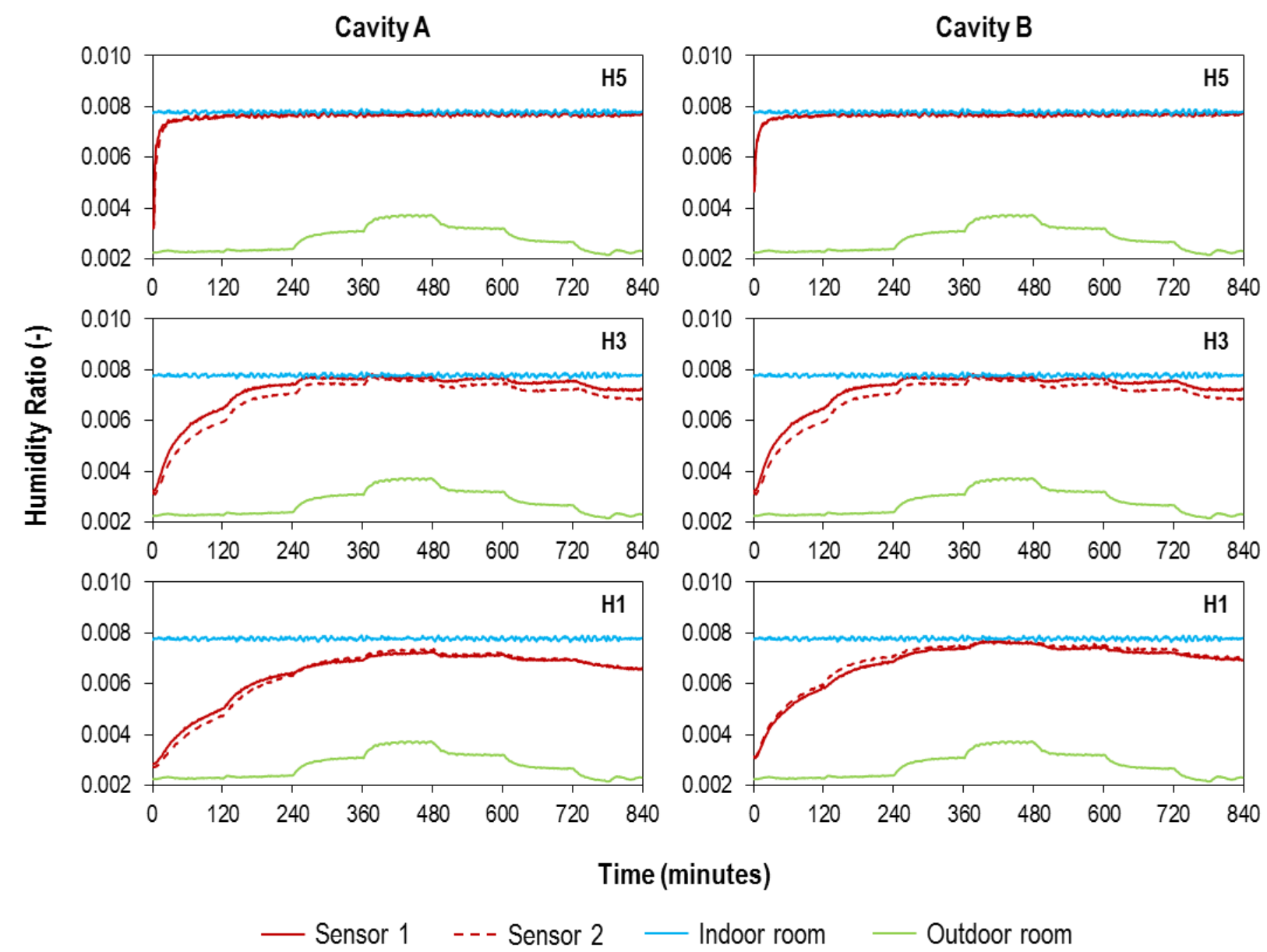

Figure A 3. Humidity ratio at exterior side of the drywall in Wall Cavities $A$ and $B$. 


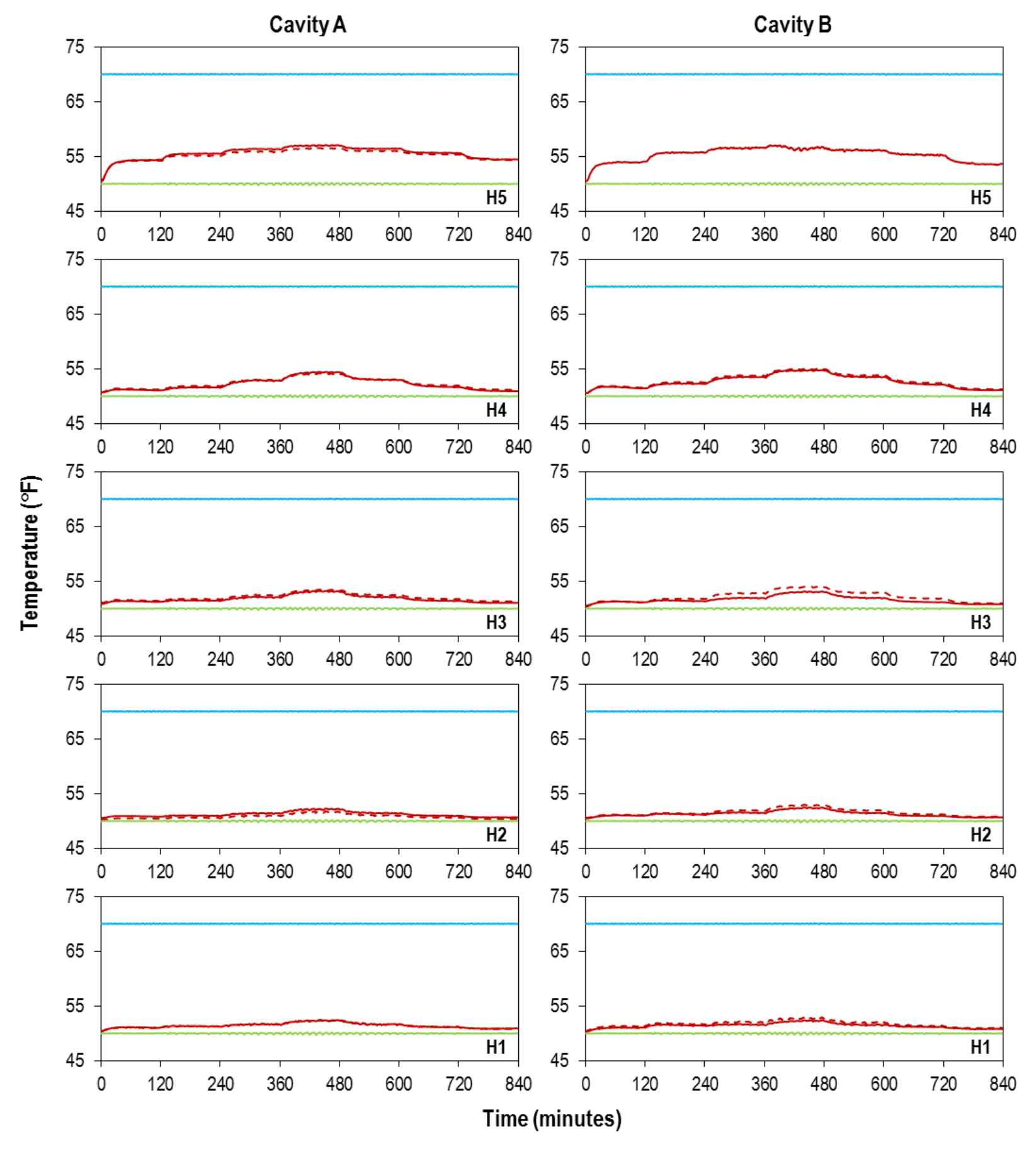

- Sensor 1 --- Sensor 2 - Indoor room - Outdoor room

Figure A 4. Temperature at exterior side of the OSB in Wall Cavities $A$ and B. 


\section{APPENDIX B: SIMULATION RESULTS}

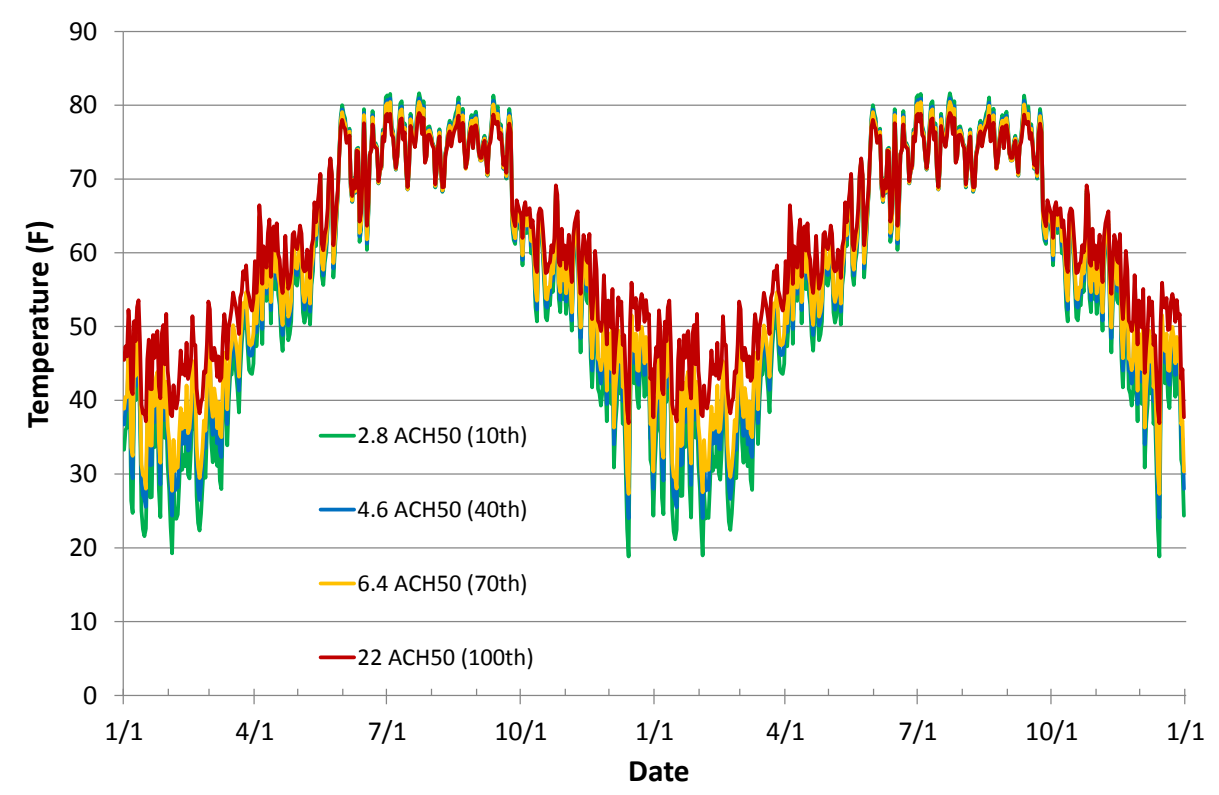

Figure B 1. Temperature at OSB near the air's entry point for a wall assembly in Climate Zone 5 and high indoor moisture. The curves represent the result from a simulated wall with four different values of airtightness (ACH50).

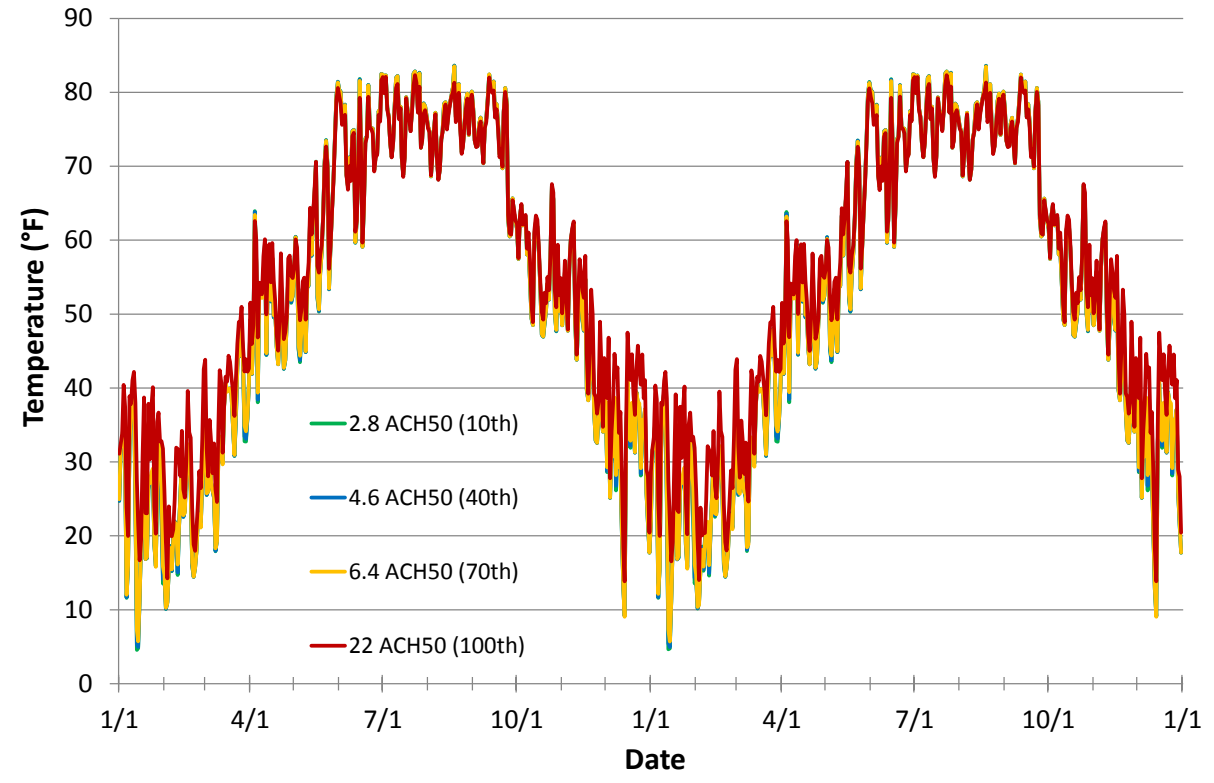

Figure $B$ 2. Temperature at OSB near the air's exit point for a wall assembly in Climate Zone 5 and high indoor moisture. The curves represent the result from a simulated wall with four different values of airtightness (ACH50). 


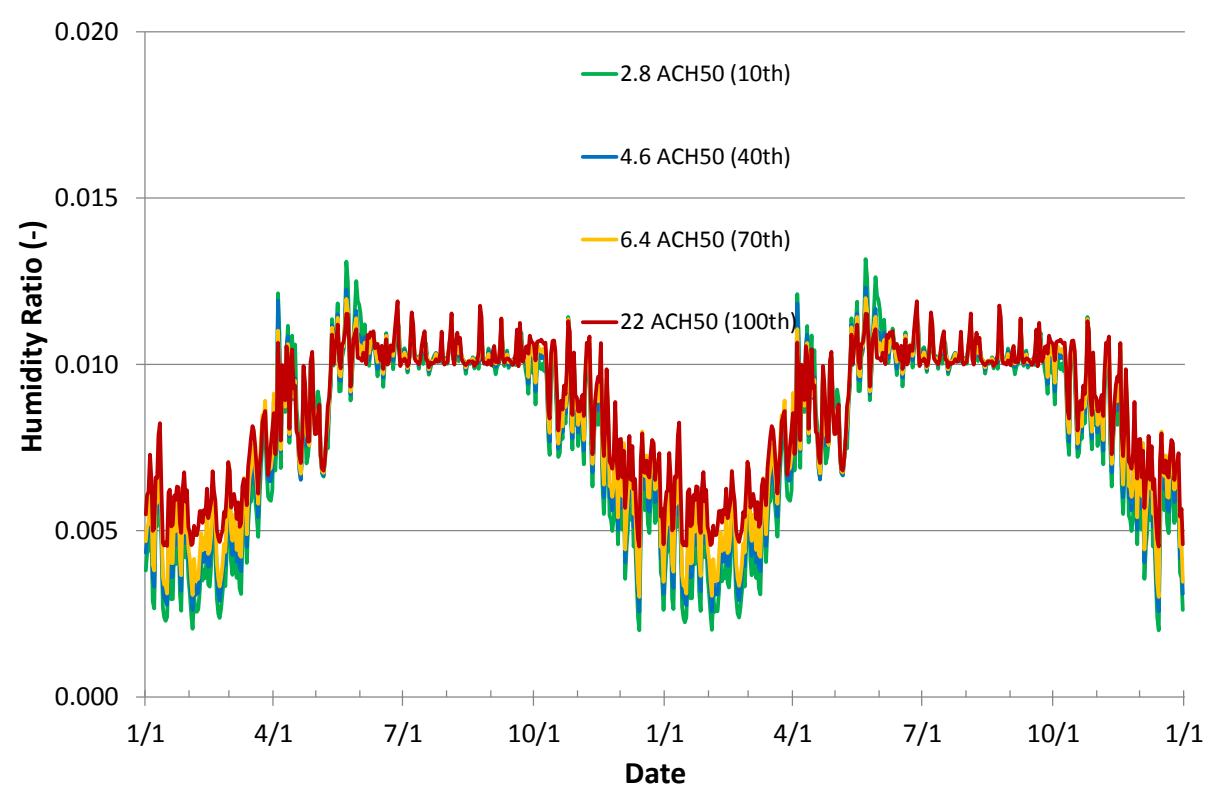

Figure B 3. Humidity ratio at OSB near the air's entry point for a wall assembly in Climate Zone 5 and high indoor moisture. The curves represent the result from a simulated wall with four different values of airtightness (ACH50).

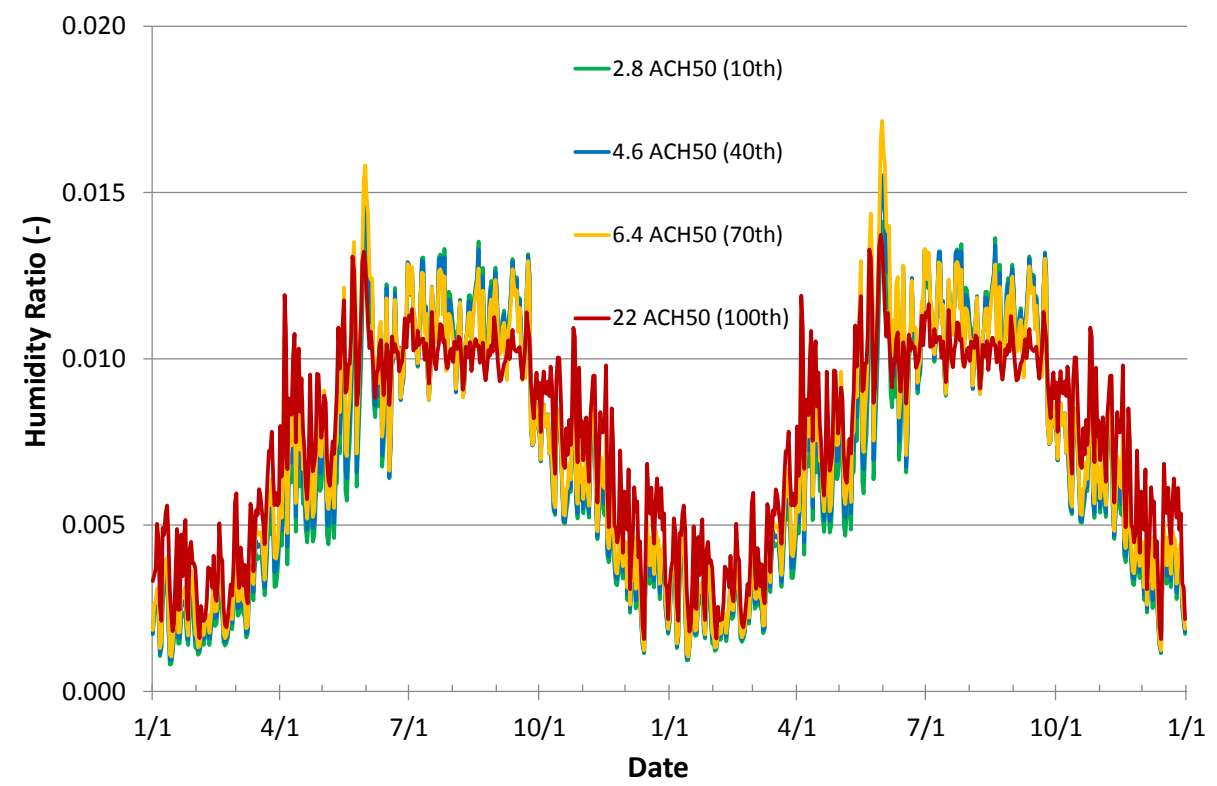

Figure B 4. Humidity ratio at OSB near the air's exit point for a wall assembly in Climate Zone 5 and high indoor moisture. The curves represent the result from a simulated wall with four different values of airtightness (ACH50). 


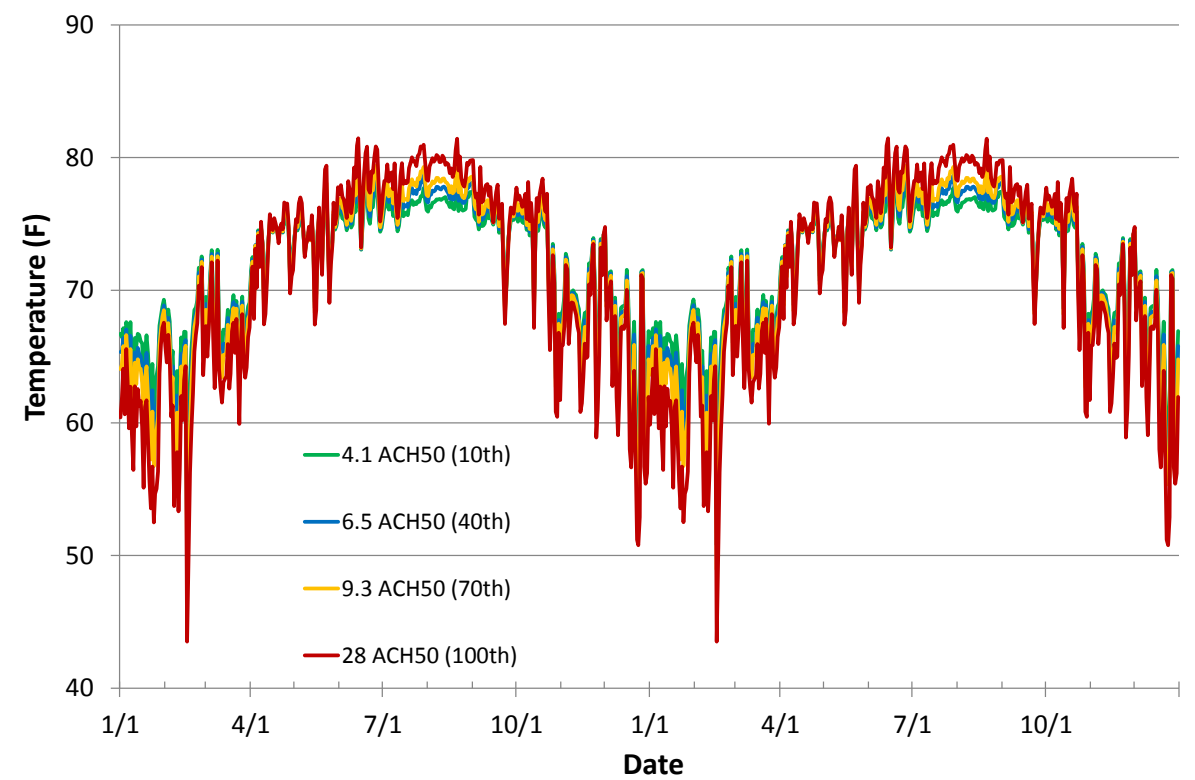

Figure B 5. Temperature at drywall near the air's entry point for a wall assembly in Climate Zone 2 and high indoor moisture. The curves represent the result from a simulated wall with four different values of airtightness (ACH50).

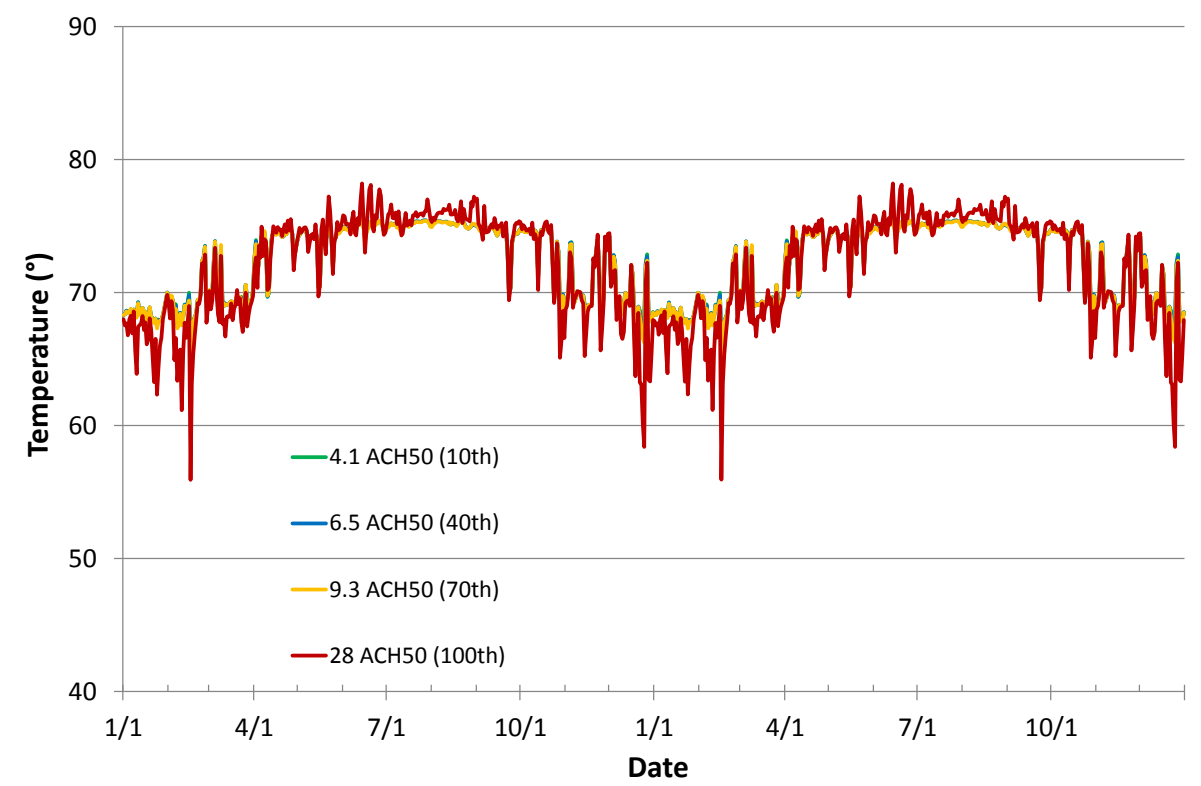

Figure B 6. Temperature at dyrwall near the air's exit point for a wall assembly in Climate Zone 2 and high indoor moisture. The curves represent the result from a simulated wall with four different values of airtightness (ACH50). 


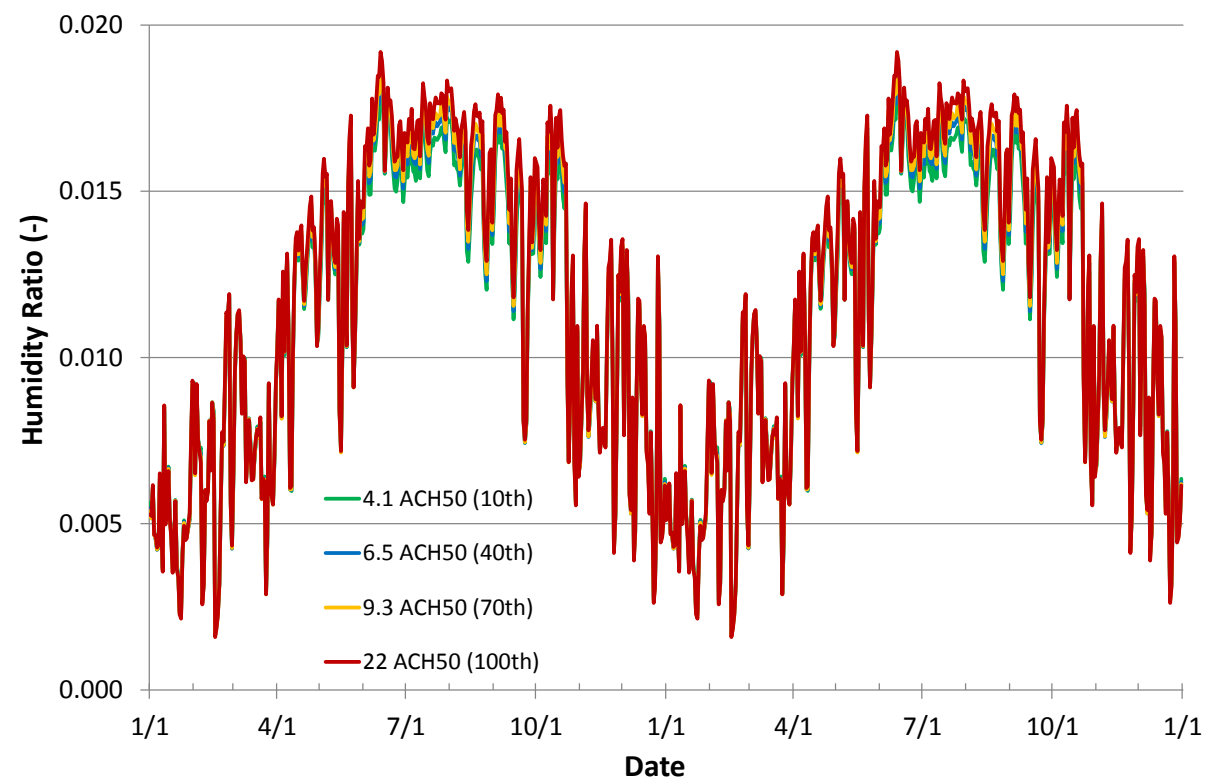

Figure B 7. Humidity ratio at drywall near the air's entry point for a wall assembly in Climate Zone 2 and high indoor moisture. The curves represent the result from a simulated wall with four different values of airtightness (ACH50).

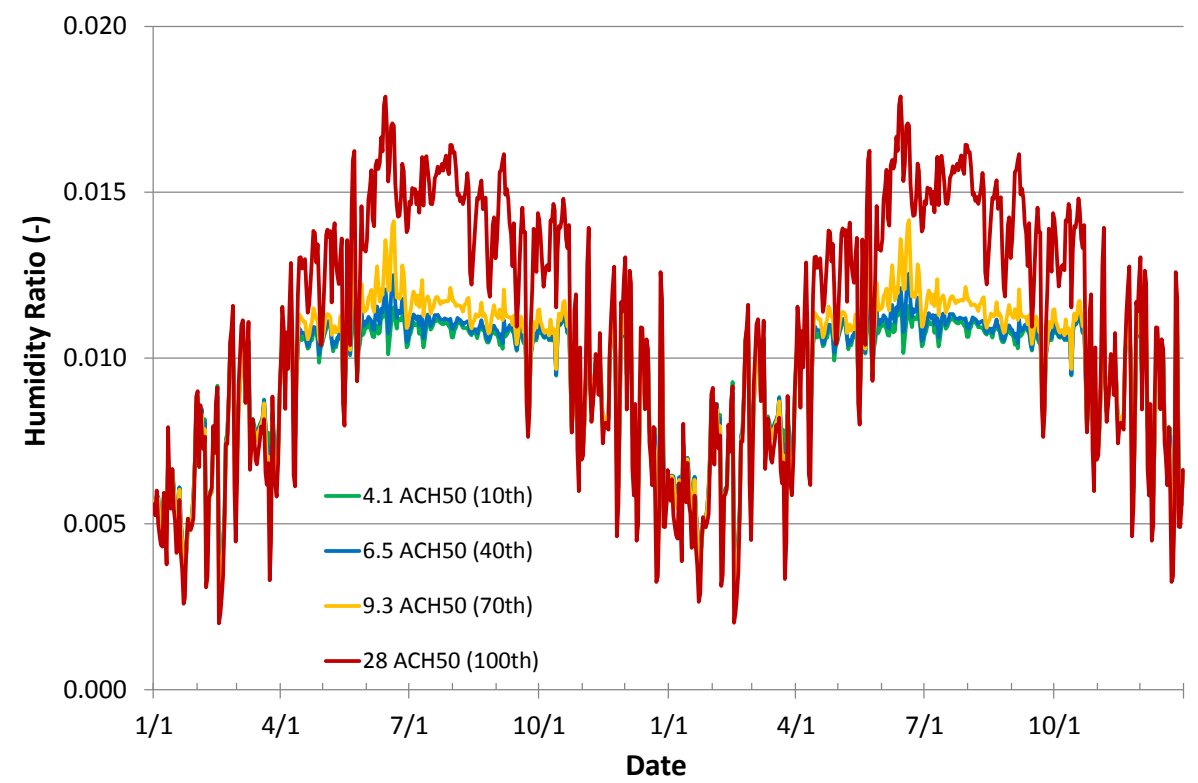

Figure B 8. Humidity ratio at drywall near the air's exit point for a wall assembly in Climate Zone 2 and high indoor moisture. The curves represent the result from a simulated wall with four different values of airtightness (ACH50). 